\title{
Periodic stability analysis of wind turbines operating in turbulent wind conditions
}

\author{
Riccardo Riva $^{1}$, Stefano Cacciola ${ }^{2}$, and Carlo Luigi Bottasso ${ }^{1,2}$ \\ ${ }^{1}$ Dipartimento di Scienze e Tecnologie Aerospaziali, Politecnico di Milano, Milano, Italy \\ ${ }^{2}$ Wind Energy Institute, Technische Universität München, Garching bei München, Germany \\ Correspondence to: Carlo Luigi Bottasso (carlo.bottasso@tum.de) \\ Received: 19 December 2015 - Published in Wind Energ. Sci. Discuss.: 21 January 2016 \\ Revised: 28 July 2016 - Accepted: 9 September 2016 - Published: 20 October 2016
}

\begin{abstract}
The formulation is model-independent, in the sense that it does not require knowledge of the equations of motion of the periodic system being analyzed, and it is applicable to an arbitrary number of blades and to any configuration of the machine. In addition, as wind turbulence can be viewed as a stochastic disturbance, the method is also applicable to real wind turbines operating in the field.

The characteristics of the new method are verified first with a simplified analytical model and then using a high-fidelity multi-body model of a multi-MW wind turbine. Results are compared with those obtained by the well-known operational modal analysis approach.
\end{abstract}

\section{Introduction}

Stability analysis can help address very practical issues, such as assessing the proximity of flutter boundaries, identifying low-damped modes, understanding the vibratory content of a machine, evaluating the effectiveness of control strategies for enhancing modal damping, detecting incipient failures, and many others. For linear time-invariant (LTI) systems, the stability analysis is a well-understood problem, and several methods are available (e.g. Hauer et al., 1990; M. O. L. Hansen et al., 2006; Murtagh and Basu, 2007). However, it is unfortunately not possible to ignore the periodic nature of wind turbines (Eggleston and Stoddard, 1987; Manwell et al., 2009). In fact, blades experience different wind conditions in their travel around the rotor disk, for example due to shears and wind misalignment, so that the aerodynamically induced damping and stiffness vary cyclically. Furthermore, the blade structural stiffness also varies periodically under the effects of its own weight, while couplings among tower and blades depend on the azimuthal position of the rotor. Additionally, the use of individual pitch control (IPC) may introduce yet a further source of periodicity in the system dynamics. The design of future, very large wind turbines, principally for the exploitation of off-shore wind resources, will highlight even further the importance of a rig- orous treatment of the periodic nature of the system when studying its stability. In fact, the system dynamics will be complicated by the hydro-elastic characteristics of the submerged - possibly floating - structure, including the excitation caused by periodic waves.

One popular approach to the stability analysis of rotors in general and of wind turbines in particular (see Hansen, 2004; Skjoldan and Hansen, 2011) is to use the multi-blade coordinate (MBC) transformation of Coleman and Feingold (1958). Given the dynamical system equations of motion, this periodic transformation expresses the model rotating degrees of freedom in a new set of coordinates, in this way achieving a significant reduction, but in general not an exact cancellation, of the periodic content of the state matrix. The remaining periodicity is typically removed by averaging, and the resulting LTI model is finally analyzed using standard time-invariant techniques.

In principle, there are at least three issues connected with any Coleman-based stability analysis approach.

- First, the level of approximation implied by the averaging of the remaining periodicity is difficult to assess and quantify a priori. In fact, to the authors' knowledge, there is no theoretical proof yet that the periodicity that remains after the application of the Coleman 
transformation is small in general nor that this approach amounts to some consistent and bounded approximation of a rigorous Floquet analysis. Given the widespread use of the Coleman transformation, and its generally excellent behavior, such a proof remains a goal very much worth pursuing but, to date, unattained.

- Second, the Coleman transformation unfortunately exists only for a number of blades greater than or equal to three. Although this is the most common wind turbine configuration nowadays, a revival of the two-bladed concept is possible.

- Third, codes implementing the Coleman transformation require access to the linearized equations of motion of the system. As a consequence, any addition to a simulation code has an impact on the associated stability analysis tool, resulting in extra software maintenance work.

Other possible approaches to the stability analysis of rotors have been formulated in the frequency domain. For example, the estimation of power spectra along with modal frequencies and damping ratios of an operating wind turbine has been addressed by Avendaño-Valencia and Fassois (2014). That paper considered several parametric and non-parametric methods and their application to experimental data, including the periodic autoregressive (PAR) model. In addition, periodic autoregressive moving average (PARMA) models have been considered by Avendaño-Valencia and Fassois (2013). Two subspace algorithms for periodic systems have been presented by Skjoldan and Bauchau (2011) and Mevel et al. (2014), one being used for numerically generated time series and the other for experimentally measured ones.

The operational modal analysis (OMA) has been extended to the periodic case (Allen et al., 2011b) by using the concept of the harmonic transfer function (HTF). In Allen et al. (2011b), the simple peak-picking method was used for extracting relevant properties from the spectra, while more specialized fitting algorithms were proposed by Allen et al. (2011a). Subsequent applications and developments can be found in Shifei and Allen (2014, 2012). Although the method is general, the estimation of the quantities of interest for a stability analysis from noisy spectra remains a somewhat delicate operation, as will be shown later on in the following pages.

In the authors' opinion, there are two desirable goals in the stability analysis of wind turbines that still need further investigation in order to be fully attained.

- First, one would like to account completely rigorously for the periodicity of such systems, without introducing approximations of unknown effects.

- Second, one would like to formulate the analysis so that it is system-independent. System independence is here intended to mean that a method can be applied to wind turbine models of arbitrary complexity and topology (e.g., any number of blades and horizontal or vertical axis) and also to real wind turbines operating in the field.

To answer these needs, Bottasso and Cacciola (2015) proposed a periodic stability analysis formulated in terms of input-output discrete-time responses. Such time histories could come from "virtual" experiments performed on a given model, from simplified ones to the more advanced contemporary comprehensive multi-body-based aero-hydro-servoelastic models. Using this approach, a reduced periodic autoregressive with exogenous input (PARX) model is first identified from a recorded response of the system and then used for conducting a stability analysis according to Floquet theory. On the practical side, this implies that the analysis respects the periodic nature of the problem. Furthermore, one can easily replace the model with a different one, without having to modify or adjust in any way the stability analysis procedure.

Although this approach attains the two goals outlined above, one of its limits is that it can not be used with measurements obtained on a real wind turbine operating in the field, since the effects of wind turbulence are not considered within the PARX model structure. To address this issue, the same approach was extended to account for the presence of turbulence (Bottasso et al., 2014). Using this new technique, one first identifies a periodic autoregressive moving average with exogenous input (PARMAX) model, whose stability is then analyzed according to Floquet. Bottasso et al. (2014) showed only one example related to the first blade edgewise mode of a wind turbine rotor. The goal of the present paper is to expand and formulate in detail the PARMAX-based method originally proposed by Bottasso et al. (2014). A second goal of this paper is to compare the PARMAX method with the periodic operational modal analysis (POMA) (see Allen et al., 2011a), which is taken here to represent the accepted state of the art for the stability analysis of wind turbines operating in turbulent wind conditions.

The article is organized according to the following plan. The problem of the identification of PARMAX models is addressed in Sect. 2. Here, a newly developed algorithm that has its basis in the prediction error method (PEM) is formulated, with particular emphasis on the guaranteed stability of the PARMAX predictor. Section 3 is devoted to POMA theory. After reviewing the concept of HTFs, the treatment proceeds by discussing the method and its use for conducting periodic stability analyses. As the authors are not aware of a reference collecting together all useful background information on Floquet theory and the signal analysis tools needed for POMA, this material is synthetically reviewed in Appendix A, to ease reading. The accuracy of the PARX and POMA identification techniques is then compared to an exact reference in Sect. 4. To this purpose, first a nonlinear wind turbine analytical model is developed. Then, the stability of 
its linearized version is studied according to Floquet theory, providing a reference ground truth used for comparing PARX and POMA. The equations of such an analytical model are derived in Appendix B. In Sect. 5, a procedure to obtain the Campbell diagram of a rotor with the PARMAX method is described. PARMAX and POMA techniques are then used to identify the first low-damped modes of a high-fidelity wind turbine model, operating in the partial load region in turbulent winds. Conclusions and recommendations are then given in the final section of the paper.

\section{The PARMAX model}

\subsection{Modeling of wind turbine behavior in turbulent wind conditions using the PARMAX sequence}

Bottasso and Cacciola (2015) showed that the relevant dynamics of a wind turbine output can be accurately captured by a PARX sequence. Stability is then verified by applying Floquet theory to the PARX reduced model. The resulting process is model-independent and fully compliant with the periodic nature of the problem. However, the use of PARX models must be restricted to systems subjected to deterministic inputs, as their structure does not consider the presence of process noise, such as atmospheric turbulence. As a step towards the application of this periodic stability analysis concept to real wind turbines, a PARMAX sequence is considered here.

In accordance with Bottasso and Cacciola (2015), the deterministic behavior of a wind-turbine-measured output $z$ can be modeled with a PARX sequence as

$\mathfrak{A}(q ; k) z(k)=\mathfrak{B}(q ; k) u_{\mathrm{t}}(k)$,

where $k$ is the time index and $q$ the back-shift operator, such that $z(k) q^{-i}=z(k-i)$. The autoregressive and exogenous parts are defined, respectively, by polynomials $\mathfrak{A}(q ; k)$ and $\mathfrak{B}(q ; k)$ as

$$
\begin{aligned}
& \mathfrak{A}(q ; k)=1-\sum_{i=1}^{N_{a}} a_{i}(k) q^{-i}, \\
& \mathfrak{B}(q ; k)=\sum_{j=0}^{N_{b}} b_{i}(k) q^{-i},
\end{aligned}
$$

both being characterized by periodic coefficients $a_{i}(k)=$ $a_{i}(k+K)$ and $b_{j}(k)=b_{j}(k+K)$, where $N_{a}$ and $N_{b}$ indicate the order of the AR and X part, respectively, while $K$ is the period of the system. Finally, $u_{\mathrm{t}}$ is the input, assumed here to be the turbulent wind.

The stochastic nature of the turbulent wind field violates the assumption of a deterministic and fully measurable input $u_{\mathrm{t}}$. To account for this, the actual wind is viewed as a sum of two distinct contributions: a mean wind $u(k)$ and a turbulence-induced perturbation $\delta u_{\mathrm{t}}(k)$. As the spectrum of the atmospheric turbulence is far from being constant, $\delta u_{\mathrm{t}}(k)$ is modeled by means of a shape filter $\mathfrak{F}(q ; k)$ such that

$u_{\mathrm{t}}(k)=u(k)+\mathfrak{F}(q ; k) e(k)$,

where $e(k)$ is a zero-mean, white, and Gaussian noise, with periodic variance $\sigma(k)^{2}$.

Inserting Eq. (3) into Eq. (1), the following is derived:

$\mathfrak{A}(q ; k) z(k)=\mathfrak{B}(q ; k) u(k)+\mathfrak{G}(q ; k) e(k)$,

where $\mathfrak{C}(q ; k)=\mathfrak{B}(q ; k) \mathfrak{F}(q ; k)$. Equation (4) is a PARMAX model whose MA part is represented by polynomial $\mathfrak{G}(q ; k)$, defined as

$\mathfrak{G}(q ; k)=1+\sum_{i=1}^{N_{g}} g_{w}(k) q^{-i}$,

where $g_{w}(k)=g_{w}(k+K)$ are the MA periodic coefficients and $N_{g}$ the MA order. The overall order of the system is defined as $n=\max \left(N_{a}, N_{b}, N_{g}\right)$. The resulting PARMAX sequence is then

$$
\begin{aligned}
& z(k)=\sum_{i=1}^{N_{a}} a_{i}(k) z(k-i)+\sum_{j=0}^{N_{b}} b_{j}(k) u(k-j) \\
& +\sum_{w=1}^{N_{g}} g_{w}(k) e(k-w)+e(k) .
\end{aligned}
$$

It should be remarked that the present approach does not consider the effects of nonlinearities nor of rotor speed variations induced by turbulence. The former potential problem can be checked a posteriori by looking at the matching between predicted and measured quantities. The latter can be partially solved by averaging the rotor speed over the analyzed time window. Typically, because of the large inertia of wind turbine rotors, angular speed variations are not expected to be highly significant, especially within the short time windows required by the proposed approach.

\subsection{State space representation of PARMAX sequences}

In order to perform a stability analysis according to Floquet theory (cf. Bottasso and Cacciola, 2015 and the review reported in Appendix A), it is necessary to realize the PARMAX Eq. (6) in an equivalent state space representation. To this end, consider a linear discrete-time system with timevarying coefficients in observable canonical form:

$$
\begin{aligned}
\boldsymbol{x}(k+1) & =\mathbf{A}(k) \boldsymbol{x}(k)+\mathbf{B}(k) u(k)+\mathbf{E}(k) e(k), \\
y(k) & =\mathbf{C}(k) \boldsymbol{x}(k)+\mathbf{D}(k) u(k)+\mathbf{F}(k) e(k),
\end{aligned}
$$

where $\boldsymbol{x}(k)=\left(x_{1}(k), \ldots, x_{n}(k)\right)^{T}$, while the system matrices are given by

$\left[\begin{array}{c|c|c}\mathbf{A}(k) & \mathbf{B}(k) & \mathbf{E}(k) \\ \mathbf{C}(k) & \mathbf{D}(k) & \mathbf{F}(k)\end{array}\right]$ 


$$
=\left[\begin{array}{ccccc|c|c}
0 & 0 & \cdots & 0 & \alpha_{n}(k) & \beta_{n}(k) & \gamma_{n}(k) \\
1 & 0 & \cdots & 0 & \alpha_{n-1}(k) & \beta_{n-1}(k) & \gamma_{n-1}(k) \\
0 & 1 & \cdots & 0 & \alpha_{n-2}(k) & \beta_{n-2}(k) & \gamma_{n-2}(k) \\
\vdots & \ddots & \ddots & \vdots & \vdots & \vdots & \vdots \\
0 & 0 & \cdots & 1 & \alpha_{1}(k) & \beta_{1}(k) & \gamma_{1}(k) \\
0 & 0 & \cdots & 0 & 1 & \beta_{0}(k) & 1
\end{array}\right] .
$$

Including the presence of the MA part, the input-output sequence of system Eq. (7) can be derived as

$$
\begin{aligned}
& y(k)=\sum_{i=1}^{n} \alpha_{i}(k-i) y(k-i) \\
& +\sum_{i=1}^{n}\left(\beta_{i}(k-i)-\beta_{0}(k-i) \alpha_{i}(k-i)\right) u(k-i)+\beta_{0} u(k) \\
& +\sum_{i=1}^{n}\left(\gamma_{i}(k-i)-\alpha_{i}(k-i)\right) e(k-i)+e(k) .
\end{aligned}
$$

Comparing Eq. (6) with Eq. (9), the following equivalence relations are obtained

$$
\begin{aligned}
& \alpha_{i}(k)=a_{i}(k+i) \quad \forall i=\left(1, \ldots, N_{a}\right), \\
& \beta_{0}(k)=b_{0}(k), \\
& \beta_{i}(k)=b_{i}(k+i)+a_{i}(k+i) b_{0}(k) \quad \forall i=\left(1, \ldots, N_{b}\right), \\
& \gamma_{i}(k)=g_{i}(k+i)+a_{i}(k+i) \quad \forall i=\left(1, \ldots, N_{g}\right),
\end{aligned}
$$

which readily give the state space system matrices. Once these are known, stability is assessed according to Floquet theory as described in Appendix A.

\subsection{Identification through the prediction error method}

In the present context, a single-input single-output (SISO) PARMAX model must be identified from a sequence of $N$ measurements. Among the plethora of existing estimation methods, which may range from time to frequency domain and from optimization-based to subspace algorithms, the PEM (Bittanti et al., 1994) is chosen here. This method has been frequently used for rotating systems, such as rotorcraft vehicles and wind turbines. For example, the periodic equation-error method was used for identifying a reducedorder model of a helicopter rotor by Bertogalli et al. (1999), whereas Bottasso and Cacciola (2015) proposed a periodic output-error method for the identification of reduced wind turbine models.

The estimation problem, formalized according to the PEM, is the one of finding the periodic coefficients $a_{i}(k)$, $b_{j}(k)$, and $g_{w}(k)$ that minimize the cost function $J$ defined as the mean value of the square of the prediction error, i.e.,

$J=\frac{1}{N} \sum_{k=1}^{N} \varepsilon^{2}(k)$.

Here $\varepsilon(k)=z(k)-\hat{z}(k \mid k-1)$ is the prediction error at time instant $k$, being $\hat{z}(k \mid k-1)$ (hereafter more concisely written as $\hat{z}(k))$, the optimal one-step-ahead prediction of $z(k)$ based on knowledge of all data until time step $k-1$. According to Bittanti and De Nicolao (1993) and Ljung (1999), the optimal one-step-ahead predictor of process (6) is

$$
\begin{aligned}
& \hat{z}(k)=-\sum_{i=1}^{n} g_{i}(k) \hat{z}(k-i) \\
& +\sum_{j=1}^{n}\left(a_{j}(k)+g_{j}(k)\right) z(k-i)+\sum_{w=1}^{n} b_{i}(k) u(k-i) .
\end{aligned}
$$

As previously argued, the presence of the MA part in the PARMAX model allows for a more adequate characterization of the process noise term, at the cost of a more complex estimation procedure. In fact, the optimal predictor of the PARMAX process expressed by Eq. (12) is nonlinear in the parameters, as any $\hat{z}(k)$ is a function of its previous values $\hat{z}(k-w)$, which in turn depend on the parameters. Consequently, the minimization of cost function Eq. (11) involves an iterative optimization. If the MA part in Eqs. (6) and (12) is neglected, a PARX sequence is obtained and the estimation problem reduces to the so-called equation-error approach (Bottasso and Cacciola, 2015; Bottasso et al., 2014).

Moreover, it is easy to verify that predictor Eq. (12) is by itself a PARX dynamic system, in which the autoregressive part is described by coefficients $-g_{w}(k)$, whereas coefficients $a_{j}(k)+g_{j}(k)$ and $b_{j}(k)$ define two X parts with inputs $z(k)$ and $u(k)$, respectively. This fact is not surprising, since it often happens that the poles of the predictor coincide with the zeros of the system to be predicted. As a consequence, it may happen that, during the optimization, coefficients $g_{w}$ define an unstable predictor, jeopardizing the entire identification process (see Bittanti and De Nicolao, 1993).

In the literature there are basically two methods to enforce the stability of the MA part. The first is a heuristic approach in which the coefficients $g_{w}(k)$ are perturbed (for example, halved) repeatedly until the achievement of a stable predictor. This method actually corresponds to a re-initialization of the parameters with unpredictable effects on the convergence of the estimation. The second approach is based on the computation of a new predictor, with different coefficients $g_{w}$ but the same autocorrelation of the unstable one. For the timeinvariant case, this new canonical model can be obtained using Bauer's algorithm (Sayed and Kailath, 2001), whereas for the periodic case it can be obtained by solving a suitable periodic Riccati equation (Bittanti and De Nicolao, 1993) or through the multivariate Rissanen factorization (Bittanti et al., 1991; Rissanen, 1973).

In this work, an alternative and original method is proposed. The stability of the predictor is enforced by a nonlinear constraint within the estimation process, and the resulting constrained optimization is performed by an interior-point algorithm (cf. Byrd et al., 2000, 1999; Waltz et al., 2006). The estimation problem is then reformulated as

$$
\boldsymbol{p}=\arg \min _{\boldsymbol{p}} J(\varepsilon(k) ; \boldsymbol{p}),
$$




$$
\text { s.t. : }|\mathcal{P}(\boldsymbol{p})|<1,
$$

where $\boldsymbol{p}$ is the vector of the unknown coefficients and $\mathcal{P}(\boldsymbol{p})$ are the characteristic multipliers of the PARMAX predictor.

The characteristic multipliers that constrain the estimation problem can be computed from the autoregressive part of Eq. (12), i.e., $\hat{y}(k)=\sum_{w}-g_{w}(k) \hat{y}(k-w)$, which can be realized as a state space form according to Eqs. (7)-(10), leading to the following dynamic matrix

$$
\mathbf{N}(k)=\left[\begin{array}{ccccc}
0 & 0 & \cdots & 0 & -g_{N_{g}}\left(k+N_{g}\right) \\
1 & 0 & \cdots & 0 & -g_{N_{g}-1}\left(k+N_{g}-1\right) \\
0 & 1 & \cdots & 0 & -g_{N_{g}-2}\left(k+N_{g}-2\right) \\
\vdots & \ddots & \ddots & \vdots & \vdots \\
0 & 0 & \cdots & 1 & -g_{(}(k+1)
\end{array}\right] .
$$

The periodic coefficients $a_{i}(k), b_{j}(k)$, and $g_{w}(k)$ are approximated by using truncated Fourier expansions, i.e.,

$a_{i}(k)=a_{i 0}+\sum_{l=1}^{N_{F_{a}}}\left(a_{i_{l}}^{c} \cos (l \psi(k))+a_{i_{l}}^{s} \sin (l \psi(k))\right)$,

$b_{j}(k)=b_{j_{0}}+\sum_{m=1}^{N_{F_{b}}}\left(b_{j_{m}}^{c} \cos (m \psi(k))+b_{j_{m}}^{s} \sin (m \psi(k))\right)$,

$g_{w}(k)=g_{w_{0}}+\sum_{r=1}^{N_{F_{g}}}\left(g_{w_{r}}^{c} \cos (r \psi(k))+g_{w_{r}}^{s} \sin (r \psi(k))\right)$,

where $\psi(k)$ is the rotor azimuth. The unknown amplitudes of such expansions are collected in the vector of parameters $\boldsymbol{p}$

$\boldsymbol{p}=\left(\ldots, a_{i 0}, a_{i l}^{c}, a_{i l}^{s}, \ldots, b_{j_{0}}, b_{j_{m}}^{c}, b_{j_{m}}^{s}, \ldots, g_{w_{0}}, g_{w_{r}}^{c}, g_{w_{r}}^{s}, \ldots\right)^{T}$,

where $i=\left(1, \ldots, N_{a}\right), j=\left(1, \ldots, N_{b}\right), w=\left(1, \ldots, N_{g}\right)$, $l=\left(1, \ldots, N_{F_{a}}\right), m=\left(1, \ldots, N_{F_{b}}\right)$, and $r=\left(1, \ldots, N_{F_{g}}\right)$, $N_{F_{a}}, N_{F_{b}}$, and $N_{F_{g}}$ being the number of Fourier harmonics of the periodic coefficients for the AR, X, and MA parts, respectively.

Due to the nonlinear behavior of the predictor, the possible presence of multiple local minima has to be taken into account. A suitable starting point for the nonlinear problem can be selected by fitting the recorded data with simpler models such as ARMAX or PARX (Bittanti et al., 1991) or by using a recursive extended least-squares algorithm (AvendañoValencia and Fassois, 2013; Spiridonakos and Fassois, 2009). In the present work, convergence to the global minimum is ensured by performing several optimization trials from a randomly chosen set of initial conditions.

\section{Theory of periodic operational modal analysis}

The OMA is an output-only system identification technique, which has been widely used to conduct modal analyses of different mechanical systems. Recently, special attention has been devoted in the literature to the application of OMA in the field of wind energy (Carne and James, 2010) and to the related underlying hypotheses (Chauhan et al., 2009; Tcherniak et al., 2010). An output-only technique specifically tailored to time periodic systems was developed by Allen et al. (2011b). This technique, called periodic OMA (POMA), exploits the particular behavior of a linear time periodic (LTP) system in the frequency domain, as described by the HTF (see Sect. A2 for details). In the present paper, POMA will be briefly reviewed and then compared to the PARMAX-based stability analysis proposed here.

Consider a strictly proper periodic system and the exponentially modulated periodic (EMP) expansions of its input and output, noted, respectively, as $\mathcal{U}$ and $\mathcal{Y}$, as described in Sect. A2. The input-output behavior of the system can be analyzed through the HTF $\mathcal{G}$ as

$\mathcal{U}(s)=\mathcal{G}(s) \mathcal{Y}(s)$,

with $s \in \mathbb{C}$ and $\mathcal{G}(s)$ defined according to Eq. (A44). Projecting Eq. (17) onto the imaginary axis, each element of the EMP expansion of $\mathcal{Y}$ and $\mathcal{U}$ can be computed as the Fourier transform of frequency-shifted copies of $y(t)$ and $u(t)$ as

$y_{k}(\omega)=\int_{-\infty}^{\infty} y(t) \mathrm{e}^{(l \omega+l k \Omega) t} \mathrm{~d} t$,
$u_{k}(\omega)=\int_{-\infty}^{\infty} u(t) \mathrm{e}^{(l \omega+l k \Omega) t} \mathrm{~d} t$.

As reported in Wereley (1991) and briefly reviewed in Allen et al. (2011b), the input-output behavior in the frequency domain can be expressed as

$\boldsymbol{Y}(\omega)=\mathbf{G}(\omega) \boldsymbol{U}(\omega)$,

where

$\boldsymbol{Y}(\omega)=\left(\begin{array}{lllll}\cdots & y_{-1}(\omega) & y_{0}(\omega) & y_{1}(\omega) & \cdots\end{array}\right)^{T}$,

$\boldsymbol{U}(\omega)=\left(\begin{array}{lllll}\cdots & u_{-1}(\omega) & u_{0}(\omega) & u_{1}(\omega) & \cdots\end{array}\right)^{T}$.

Accordingly, the harmonic frequency response function (HFRF) $\mathbf{G}(\omega)$ is given by

$\mathbf{G}(\omega)=\sum_{j=1}^{N_{\mathrm{s}}} \sum_{w=-\infty}^{\infty} \frac{\overline{\mathbf{C}}_{j, w} \overline{\mathbf{B}}_{j, w}^{T}}{\imath \omega-\left(\eta_{j}+\imath w \Omega\right)}$,

where $\overline{\mathbf{C}}_{j, w}$ and $\overline{\mathbf{C}}_{j, w}$ are defined in Eqs. (A45) and (A46) of Sect. A2.

The power spectrum of the output, noted as $\mathbf{S}_{Y Y}(\omega)$, can be written in terms of the HFRF $\mathbf{G}(\omega)$ and the power spectrum of the input $\mathbf{S}_{U U}(\omega)$ as

$\mathbf{S}_{Y Y}(\omega)=\mathbf{G}(\omega) \mathbf{S}_{U U}(\omega) \mathbf{G}(\omega)^{H}$, 
where $(\cdot)^{H}$ denotes the complex-conjugate transpose. Inserting Eqs. (21) into (22), the following expression is derived:

$$
\begin{gathered}
\mathbf{S}_{Y Y}(\omega)=\sum_{j=1}^{N_{\mathrm{s}}} \sum_{w=-\infty}^{\infty} \sum_{p=1}^{N_{\mathrm{s}}} \sum_{q=-\infty}^{\infty} \\
\frac{\overline{\mathbf{C}}_{j, w} \mathbf{W}(\omega)_{j, w, p, q} \overline{\mathbf{C}}_{p, q}^{H}}{\left(\iota \omega-\left(\eta_{j}+\imath w \Omega\right)\right)\left(\iota \omega-\left(\eta_{p}+\imath q \Omega\right)\right)^{H}},
\end{gathered}
$$

where $\mathbf{W}_{j, w, r, t}=\overline{\mathbf{B}}_{j, r} \mathbf{S}_{U U} \overline{\mathbf{B}}_{w, t}^{H}$. Equation (23) can be simplified first by considering a flat expanded input power spectrum $\mathbf{W}_{j, r}(\omega)=\overline{\mathbf{B}}_{j, r} \mathbf{S}_{U U} \overline{\mathbf{B}}_{j, r}^{H}$, at least in the band of interest of a specific mode, and secondly by assuming that all modes of the system are "suitably separated".

The first requirement was analyzed extensively for wind turbine problems in Tcherniak et al. (2010). There the authors pointed out that the extended input spectrum could be significantly colored, a problem that requires particular care with simplified output-only methods. The second requirement deserves special attention as well. In fact, not only is the separation of the principal harmonics of two modes required, but it is also necessary that all super-harmonics with significant participation are well separated. For rotary wing systems, this requirement has to be considered especially carefully when looking at the whirling modes, as the principal harmonics of backward and forward modes are typically separated by about $2 \Omega$. This typically creates a crisscrossing of modes in the frequency-rotor-speed plane, leading to frequent frequency encounters.

If such conditions are verified, the extended input spectrum $\mathbf{W}$ loses its dependency on $\omega$, and the contribution of mode $\eta_{p}+\imath q \Omega$ to mode $\eta_{j}+\imath w \Omega$ can be neglected when $p \neq j$ and $q \neq w$. Hence, Eq. (23) is simplified to

$$
\begin{aligned}
& \mathbf{S}_{Y Y}(\omega) \approx \sum_{j=1}^{N_{\mathrm{S}}} \sum_{w=-\infty}^{+\infty} \\
& \frac{\overline{\mathbf{C}}_{j, w} \mathbf{W}_{j, w} \overline{\mathbf{C}}_{j, w}^{H}}{\left(\iota \omega-\left(\eta_{j}+\imath w \Omega\right)\right)\left(\iota \omega-\left(\eta_{j}+\imath w \Omega\right)\right)^{H}} .
\end{aligned}
$$

From Eq. (24) one can see that the peak related to any superharmonic of a given mode can be viewed as the peak of a linear time-invariant mode. Accordingly, one is allowed to use a standard LTI frequency domain identification technique (e.g., peak picking, curve fitting) to compute frequencies, damping factors, and modal shapes from the measured spectra.

Moreover, neglecting again the contribution of overlapping modes, one can also estimate the participation by evaluating the power spectra at the peak frequency, since

$\overline{\mathbf{C}}_{j, w} \overline{\mathbf{C}}_{j, w}^{H} \propto \mathbf{S}_{\boldsymbol{Y} \boldsymbol{Y}}\left(\omega_{j}+w \Omega\right)$.
Expressing the product $\overline{\mathbf{C}}_{j, w} \overline{\mathbf{C}}_{j, w}^{H}$, one gets

$$
\overline{\mathbf{C}}_{j, w} \overline{\mathbf{C}}_{j, w}^{H}=\left[\begin{array}{ccccc}
\ddots & \vdots & \vdots & \vdots & . \\
\cdots & c_{j_{-2}} c_{j_{-1}}^{*} & c_{j_{-1}} c_{j_{0}}^{*} & c_{j_{0}} c_{j_{1}}^{*} & \cdots \\
\cdots & c_{j_{-1}} c_{j-1}^{*} & c_{j 0} c_{j_{0}}^{*} & c_{j_{1}} c_{j_{1}}^{*} & \cdots \\
\cdots & c_{j_{0}} c_{j-1}^{*} & c_{j_{1}} c_{j_{0}}^{*} & c_{j_{2}} c_{j_{1}}^{*} & \cdots \\
\vdots & \vdots & \vdots & \vdots & \ddots
\end{array}\right],
$$

$(\cdot)^{*}$ being the complex conjugate. From Eq. (26), one could envision several criteria for extracting the participation factors for each harmonic belonging to the $j$ th mode. The simplest one is to compute the central column of the HTF and to pick the amplitudes of the spectra at the frequency of interest. The participation factors are then extracted according to Eq. (A27), reported in Sect. A1, as

$\phi_{j_{n}}^{y}=\frac{\left|c_{j_{n}}\right|}{\sum_{n}\left|c_{j_{n}}\right|}=\frac{\left|c_{j_{n}}\right|\left|c_{j_{0}}^{*}\right|}{\sum_{n}\left|c_{j_{n}}\right|\left|c_{j_{0}}^{*}\right|}=\frac{\left|c_{j_{n}} c_{j_{0}}^{*}\right|}{\sum_{n}\left|c_{j_{n}} c_{j_{0}}^{*}\right|}$.

One can also perform multiple estimations of the participation factors by looking again at the central column of $\mathbf{S}_{Y Y}$. In fact, from Eq. (26), it appears that the amplitudes picked from the $\ell$ th column at frequency $\omega_{j}+w \Omega$ are equivalent to those picked from the central column at $\omega_{j}+(w+\ell) \Omega$. This also means that computing the central column could be sufficient for having an estimation of frequencies, damping, and participation factors, as already noted in Shifei and Allen (2012).

The POMA technique can then be summarized as follows:

- Compute the Fourier transforms of the frequencyshifted copies of the recorded output $y(t), y_{k}(\omega)=$ FFT $\left(y(t) \mathrm{e}^{-l k \Omega t}\right)$ and collect them in vector $\boldsymbol{Y}(\omega)=$ $\left(\ldots, y_{k}(\omega), \ldots\right)^{T}$.

- Compute the autospectrum $\mathbf{S}_{Y Y}(\omega)$ using a standard frequency domain analysis method; in the present paper the method of Welch was employed for this purpose.

- Extract the related natural frequency and damping factors from each peak present in $\mathbf{S}_{Y Y}(\omega)$ using any standard LTI frequency domain estimation tool (Allen and Ginsberg, 2006). In this paper the straightforward peakpicking method was used, as also done by Allen et al. (2011b).

- Reconstruct the Fourier coefficients $c_{j_{n}}$, and in turn the participation factors, by evaluating the spectrum in correspondence to each peak.

It is possible to restrict the analysis to the right-half plane just by noting that

$y_{n}(-\omega)=y_{-n}^{*}(\omega)$. 


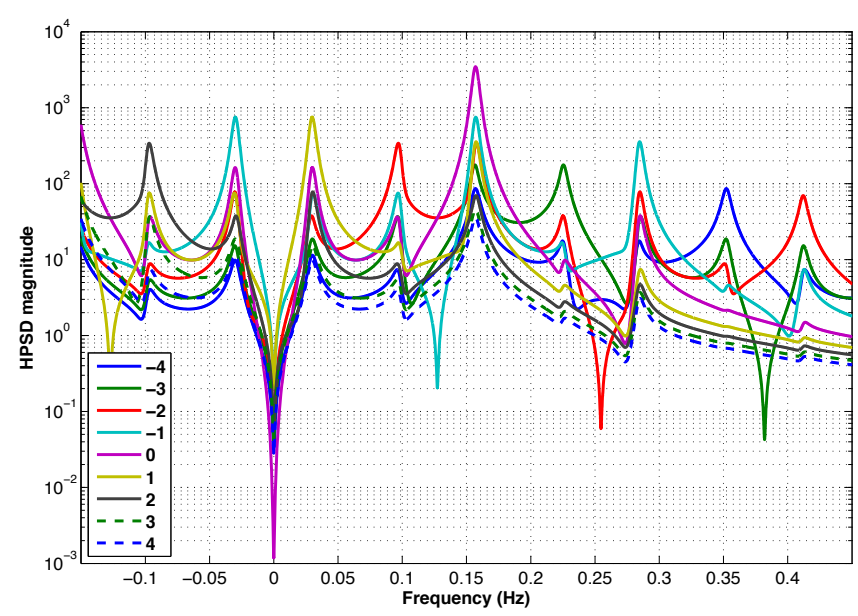

Figure 1. Harmonic power spectrum of the output of the Mathieu oscillator.

Equation (28) is particularly useful for identifying the Fourier coefficients from the peaks of the "reflected superharmonics", since according to Eq. (28) one can demonstrate that

$$
\left.c_{j_{n}}\right|_{\text {correct peak }}=\left.c_{j-n}^{*}\right|_{\text {reflected peak }} .
$$

\subsection{Application of periodic operational modal analysis to the Mathieu oscillator}

As the actual use of POMA and the correct interpretation of all peaks is not a straightforward exercise in general, a simple Mathieu oscillator is analyzed here in preparation for the application of this method to the wind turbine problems studied later on. The dynamics of a Mathieu oscillator is governed by the following equations:

$$
\begin{aligned}
\left(\begin{array}{c}
\dot{x} \\
\ddot{x}
\end{array}\right) & =\left[\begin{array}{cc}
0 & 1 \\
-\omega_{0}^{2}-\omega_{1}^{2} \cos (\Omega t) & -2 \xi \omega_{0}
\end{array}\right]\left(\begin{array}{c}
x \\
\dot{x}
\end{array}\right), \\
y & =\left[\begin{array}{ll}
1 & 0
\end{array}\right]\left(\begin{array}{c}
x \\
\dot{x}
\end{array}\right) .
\end{aligned}
$$

The parameters in Eq. (30) were set, following Allen et al. (2011b), as $\omega_{0}^{2}=1, \omega_{1}^{2}=0.4, \xi=0.04$, and $\Omega=0.8$. The system was numerically integrated from $\boldsymbol{x}(0)=(1000,0)^{T}$, and studied by means of POMA. The results were then compared with those obtained by the full Floquet theory described in Sect. A1.

Figure 1 shows the power spectra of the central column of $\mathbf{S}_{Y Y}, y_{k}(\omega) y_{0}^{H}(\omega)$ for $k=-4, \ldots, 4$. The fundamental peak (i.e., the highest one) is found on the 0 -shift curve at $0.16 \mathrm{~Hz}$ and corresponds to the amplitude $c_{j_{0}} c_{j_{0}}^{H}$. At such a frequency, all curves show a prominent peak, from which one may also easily compute the damping factors using, for example, the standard half-power bandwidth method. The participation factors are then extracted by looking at the amplitudes of the power spectra using Eq. (27).
Table 1. Frequencies and damping factors for the Mathieu oscillator and analytical results.

\begin{tabular}{ccc|cc}
\hline & \multicolumn{2}{c|}{ Frequencies } & \multicolumn{2}{c}{ Damping factors } \\
\cline { 2 - 5 } Peak & Identified & Exact & Identified & Exact \\
\hline$-4 \Omega$ & 0.3523 & 0.3523 & 0.0156 & 0.0090 \\
$-3 \Omega$ & 0.2254 & 0.2250 & 0.0220 & 0.0142 \\
$-2 \Omega$ & 0.0969 & 0.0977 & 0.0363 & 0.0326 \\
$-1 \Omega$ & 0.0299 & 0.0299 & 0.1071 & 0.1065 \\
0 & 0.1571 & 0.1571 & 0.0203 & 0.0203 \\
$+1 \Omega$ & 0.2848 & 0.2844 & 0.0114 & 0.0112 \\
$+2 \Omega$ & 0.4121 & 0.4117 & 0.0124 & 0.0077 \\
$+3 \Omega$ & 0.5390 & 0.5390 & 0.0102 & 0.0059 \\
$+4 \Omega$ & 0.6663 & 0.6664 & 0.0083 & 0.0048 \\
\hline
\end{tabular}

Starting from this peak and moving to the right, the subsequent higher peaks are found on the negative-shift curves, first in the -1 -shift one at $0.28 \mathrm{~Hz}$ and then in the -2 shift one at $0.41 \mathrm{~Hz}$, etc. The opposite happens when moving to the left. Peaks located at negative frequencies appear as reflected in the positive frequency range but with opposite shifts. This is clear if one looks at the peak located at $-0.10 \mathrm{~Hz}$, which has the -2 -shift curve as the one with the highest amplitude, whereas the reflected peak at $0.10 \mathrm{~Hz}$ is associated with the 2-shift curve. This complex behavior is easily explained by means of Eq. (28), which also states that the information in the negative frequency range can be reconstructed by looking at the curve with the opposite shift in the positive frequency plane.

Frequencies and damping factors computed from such spectra using the peak-picking method are reported in Table 1 . The same table also displays the results obtained from the full Floquet analysis of the system. The comparison shows good accuracy, especially for frequencies and damping factors of the first highest super-harmonics.

The output-specific participation factors are displayed in Table 2. Multiple estimates have been computed from each spectrum peak in the positive frequency plane. The last column also shows the analytical results. As expected, in general super-harmonics with lower participation factors are associated with higher estimation errors.

\section{Stability analysis of a model wind turbine problem}

Next, a simplified wind turbine model is used for comparing the results obtained with the PARX and POMA approaches. This is useful because it gives a way of comparing the basic performance of the two methods with respect to a known exact ground truth in the ideal case of zero disturbances. Later on in this work, the two methods will be compared for the case of a higher-fidelity wind turbine model operating in turbulent wind conditions. As no exact solution is known in that case, the preliminary investigation of this section serves the 
Table 2. Most relevant output-specific participation factors for the Mathieu oscillator and related analytical results.

\begin{tabular}{ccccccccccc}
\hline & $\begin{array}{c}0.35 \mathrm{~Hz} \\
(-4 \Omega)\end{array}$ & $\begin{array}{c}0.23 \mathrm{~Hz} \\
(-3 \Omega)\end{array}$ & $\begin{array}{c}0.10 \mathrm{~Hz} \\
(-2 \Omega)\end{array}$ & $\begin{array}{c}0.03 \mathrm{~Hz} \\
(-1 \Omega)\end{array}$ & $\begin{array}{c}\text { Peak at } \\
0.16 \mathrm{~Hz}\end{array}$ & $\begin{array}{c}0.28 \mathrm{~Hz} \\
(+1 \Omega)\end{array}$ & $\begin{array}{c}0.41 \mathrm{~Hz} \\
(+2 \Omega)\end{array}$ & $\begin{array}{c}0.54 \mathrm{~Hz} \\
(+3 \Omega)\end{array}$ & $\begin{array}{c}0.67 \mathrm{~Hz} \\
(+4 \Omega)\end{array}$ & Exact \\
\hline$\phi_{1-4}^{x}$ & 0.0174 & 0.0167 & 0.0164 & 0.0162 & 0.0163 & - & - & - & - & $4.961 \times 10^{-4}$ \\
$\phi_{1-3}^{x}$ & 0.0352 & 0.0346 & 0.0316 & 0.0323 & 0.0328 & 0.0323 & - & - & - & 0.0097 \\
$\phi_{1-2}^{x}$ & 0.0659 & 0.0587 & 0.0660 & 0.0626 & 0.0618 & 0.0652 & 0.0667 & - & - & 0.0477 \\
$\phi_{1-1}^{x}$ & 0.1509 & 0.1405 & 0.1419 & 0.1409 & 0.1410 & 0.1433 & 0.1473 & 0.1560 & - & 0.1583 \\
$\phi_{1_{0}}^{x}$ & 0.7000 & 0.6614 & 0.6445 & 0.6499 & 0.6537 & 0.6562 & 0.6753 & 0.7234 & 0.8527 & 0.7160 \\
$\phi_{1_{1}}^{x}$ & - & 0.0642 & 0.0687 & 0.0666 & 0.0661 & 0.0685 & 0.0703 & 0.0731 & 0.0862 & 0.0655 \\
$\phi_{1_{2}}^{x}$ & - & - & 0.0134 & 0.0131 & 0.0130 & 0.0134 & 0.0137 & 0.0144 & 0.0170 & 0.0023 \\
$\phi_{1_{3}}^{x}$ & - & - & - & 0.0085 & 0.0085 & 0.0086 & 0.0089 & 0.0095 & 0.0111 & $4.401 \times 10^{-5}$ \\
$\phi_{1_{4}}^{x}$ & - & - & - & - & 0.0067 & 0.0067 & 0.0069 & 0.0074 & 0.0087 & $5.325 \times 10^{-7}$ \\
\hline
\end{tabular}

purpose of clarifying whether significant differences exists between the two approaches even at this more fundamental level. Indeed, it will be shown here that some of the underlying hypotheses of POMA are not always fulfilled, and this leads occasionally to some imprecisions in the estimates of the modal quantities of interest.

The analytical model is derived in detail in Appendix B, which also gives a schematic sketch of the system in Fig. B1. The model considers the coupled motion of tower and blades subjected to aerodynamic and gravitational forces. The foreaft and side-side flexibility of the tower is rendered by two equivalent linear springs, whereas each blade is represented as a rigid body connected to the hub through two coincident linear torsional springs, allowing, respectively, the blade flapwise and edgewise rotations. The characteristics of each element in the model are chosen so as to match the first tower fore-aft and side-side modes and the first blade flap-wise and edgewise modes in vacuo of a reference $6 \mathrm{MW}$ wind turbine, as computed using a high-fidelity multi-body model. The aerodynamic formulation is inspired by the treatment of Eggleston and Stoddard (1987), in which the aerodynamic forces and moments at the blade hinges are computed assuming linear aerodynamics, small flap and lag angles, uniform inflow over the rotor disk, and constant rotor speed. The aerodynamic forces induced by tower motion, not present in the treatment of Eggleston and Stoddard (1987), are additionally considered in this paper. The model represents the complete lower spectrum of a wind turbine, including the first sideside and fore-aft tower modes, the first in-plane and out-ofplane blade modes as well as their related whirling modes.

After having collected all degrees of freedom in vector $\xi=\left(\beta_{1}, \ldots, \beta_{B}, \zeta_{1}, \ldots, \zeta_{B}, y_{H}, z_{H}\right)^{T}, B$ being the number of blades, and the inputs in vector $\boldsymbol{v}=\left(\theta_{p_{1}}, \ldots, \theta_{p_{B}}\right)^{T}, \theta_{k}$ being the pitch angle of the $k$ th blade, the resulting nonlinear second-order implicit system writes

$\boldsymbol{f}(\boldsymbol{\xi}, \dot{\boldsymbol{\xi}}, \ddot{\boldsymbol{\xi}}, \boldsymbol{v}, t)=0$.
System Eq. (31) can be integrated in time using any suitable numerical scheme, starting from a consistent set of initial conditions. This was done for generating the time histories used for PARX and POMA, paying attention not to excite the system nonlinearities, as the reference solution is based on the Floquet analysis of the linearized problem.

Since any mechanical system is linear in $\ddot{\xi}$, one may compute the mass matrix $\widehat{\mathbf{M}}(\boldsymbol{\xi}, \dot{\boldsymbol{\xi}}, t)$ and rewrite the system as $\widehat{\mathbf{M}}(\boldsymbol{\xi}, \dot{\boldsymbol{\xi}}, t) \ddot{\boldsymbol{\xi}}=\boldsymbol{g}(\boldsymbol{\xi}, \dot{\boldsymbol{\xi}}, \boldsymbol{v}, t)$. System Eq. (31), if asymptotically stable, converges to a periodic trajectory $\widetilde{\xi}(t)$ when subjected to a periodic input $\widetilde{\boldsymbol{v}}(t)$. In such a regime, the linearized periodic equations of motion write

$\mathbf{M}(t) \ddot{\hat{\boldsymbol{\xi}}}+\mathbf{T}(t) \dot{\hat{\boldsymbol{\xi}}}+\mathbf{K}(t) \hat{\boldsymbol{\xi}}+\mathbf{W}(t) \hat{\boldsymbol{v}}=0$

where the new state $\hat{\boldsymbol{\xi}}(t)$ and input $\hat{\boldsymbol{v}}(t)$ are defined as

$\hat{\boldsymbol{\xi}}(t)=\boldsymbol{\xi}(t)-\widetilde{\boldsymbol{\xi}}(t), \quad \hat{\boldsymbol{v}}(t)=\boldsymbol{v}(t)-\widetilde{\boldsymbol{v}}(t)$,

and the periodic mass, damping, stiffness, and input matrices are defined as

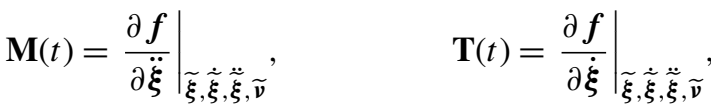

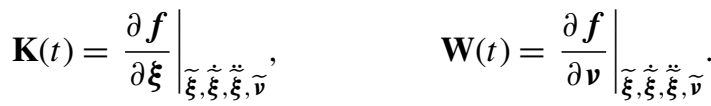

Note that $\mathbf{M}(t)$ is equal to $\widehat{\mathbf{M}}(\boldsymbol{\xi}, \dot{\boldsymbol{\xi}}, t)$, evaluated on the periodic trajectory $\widetilde{\xi}$. These linearized equations of motion about a periodic orbit were then used for developing the analysis according to Floquet, yielding the ground truth solution.

\subsection{Stability analysis of a wind turbine analytical model}

The parameters of the wind turbine analytical model loosely represent a conceptual $6 \mathrm{MW}$ wind turbine, and they are listed in Table 3. The stability of the model is studied in a uniform axial wind of $9 \mathrm{~m} \mathrm{~s}^{-1}$ for a collective pitch angle of $-0.54^{\circ}$, corresponding to operation towards the end of the partial load region. 
Table 3. Parameters of the analytical wind turbine model.

\begin{tabular}{lll}
\hline Parameter & Symbol & Value \\
\hline Number of blades & $B$ & 3 \\
Rotor radius & $R$ & $75 \mathrm{~m}$ \\
Rotor speed & $\Omega$ & $11.5 \mathrm{rpm}$ \\
Hinge offset & $e$ & $25.651 \% \mathrm{R}$ \\
Mass of hub & $m_{\mathrm{H}}$ & $7.500 \times 10^{4} \mathrm{~kg}$ \\
Blade mass (movable part) & $m_{D}$ & $1.448 \times 10^{4} \mathrm{~kg}$ \\
Blade mass (fixed part) & $m_{U}$ & $1.087 \times 10^{4} \mathrm{~kg}$ \\
Blade CG after hinge & $r_{\mathrm{GD}}$ & $18.72 \mathrm{~m}$ \\
Blade moment of inertia & $J_{D}$ & $7.488 \times 10^{6} \mathrm{~kg} \mathrm{~m}^{2}$ \\
Edgewise spring stiffness & $K_{\zeta}$ & $2.119 \times 10^{8} \mathrm{~N} \mathrm{~m}^{6}$ \\
Edgewise spring damper & $C_{\zeta}$ & $1.756 \times 10^{6} \mathrm{~N} \mathrm{~m} \mathrm{~s}^{7}$ \\
Flap-wise spring stiffness & $K_{\beta}$ & $5.215 \times 10^{7} \mathrm{~N} \mathrm{~m}$ \\
Flap-wise spring damper & $C_{\beta}$ & $1.756 \times 10^{6} \mathrm{~N} \mathrm{~m} \mathrm{~s}^{-1}$ \\
Tower SS spring stiffness & $K_{y}$ & $7.312 \times 10^{5} \mathrm{~N} \mathrm{~m}^{-1}$ \\
Tower SS spring damper & $C_{y}$ & $1.329 \times 10^{4} \mathrm{~N} \mathrm{~s} \mathrm{~m}^{-1}$ \\
Tower FA spring stiffness & $K_{z}$ & $6.581 \times 10^{5} \mathrm{~N} \mathrm{~m}^{-1}$ \\
Tower FA spring damper & $C_{z}$ & $1.329 \times 10^{4} \mathrm{~N} \mathrm{~s} \mathrm{~m}^{-1}$ \\
Lock number & $\gamma$ & 20 \\
Wind shear gradient & $K_{1}$ & $0.018 \mathrm{~s}^{-1}$ \\
\hline
\end{tabular}

The linearized periodic system was first studied using Floquet theory (see Appendix A) in order to get the exact natural frequencies, damping, and output-specific participation factors. Next, the model was used for generating all outputs needed for performing the PAR(MA)X and POMA analyses by integrating the system forward in time starting from suitable initial non-zero conditions, chosen in order to excite the modes of interest. In this exercise, the wind was considered as stationary, so that the PARMAX identification reduces to the simpler PARX one as the MA part is not necessary.

Both PARX and POMA estimates were compared with the full Floquet results in terms of relative errors for frequencies and damping factors and absolute errors for participation factors. Relative errors are defined as $v_{\mathrm{E}} / v_{\mathrm{R}}-1$, while absolute errors are defined as $v_{\mathrm{E}}-v_{\mathrm{R}}$, where $v$ is a specific modal parameter and the subscripts $\mathrm{E}$ and $\mathrm{R}$ refer, respectively, to an estimated and a real (exact) quantity.

\subsubsection{Identification of the blade edgewise mode}

The blade edgewise mode was excited by imposing the initial edgewise angles of all blades equal to a unique non-zero value, whilst all other states were set to zero at the initial time. This way the blade in-plane mode was excited while avoiding the onset of the whirling modes.

Considering first the POMA approach, the harmonic power spectrum for the second blade edgewise angle, $\zeta_{2}$, was computed with frequency shifts from $-2 \Omega$ to $+2 \Omega$. The results obtained this way are reported in Fig. 2.

Clearly, the 0-shift PSD shows a prominent peak at $\omega_{\mathrm{E}}=$ $0.86 \mathrm{~Hz}$, related to the blade in-plane mode, from which one

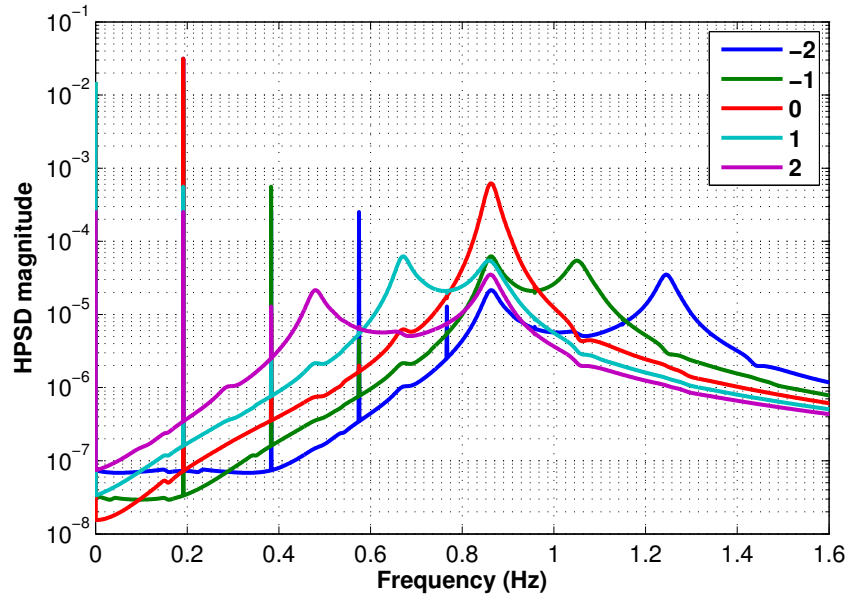

Figure 2. Harmonic power spectrum of the $\zeta_{2}$ output of the wind turbine analytical model. The peak of the $n=0$ curve is caused by the blade in-plane mode, while spikes are due to the rotational frequency and its multiples.

may easily extract the frequency and damping factor of the principal harmonic. The peak-picking method could in principle be applied to any of the peaks displayed in the figure; however, one may observe that most of the peaks are of a low amplitude and often barely noticeable from the side band of the principal harmonic. For example, the super-harmonic at $0.67 \mathrm{~Hz}$, even if visible within the 0 -shift curve, does not have enough energy to allow one to estimate its modal quantities to any reasonable accuracy. Therefore, it was preferred to compute frequency and damping factors only by looking at the highest peaks: the frequency and damping factor of the super-harmonic at $\omega_{\mathrm{E}}+\Omega$ were extracted from the peak at $1.05 \mathrm{~Hz}$ of the -1 -shift curve, while those of the super-harmonic at $\omega_{\mathrm{E}}+2 \Omega$ were extracted from the peak at $1.24 \mathrm{~Hz}$ of -2 -shift curve, and similarly for the other superharmonics. For the same reason, participation factors were obtained only by looking at the PSD amplitude at $\omega_{\mathrm{E}}$. In fact, at this frequency all curves show peaks that are prominent and distinct enough to compute the participation factors according to Eq. (27).

Next, the PARX analysis was considered. As long as only the blade in-plane mode is significantly excited, as indicated from the 0-shift curve in Fig. 2, the order of the AR part may be set as $N_{a}=2$. A first-order X part $\left(N_{b}=1\right)$ was considered as the inputs (wind speed and pitch angle) are constant in this case. Finally, the number of harmonics for the Fourier series expansion of both the $\mathrm{AR}$ and $\mathrm{X}$ parts, $N_{F_{a}}$ and $N_{F_{b}}$, were both set equal to 1 . The matching between predicted and simulated output, not reported here for the sake of brevity, showed excellent correlation, proof of the fact that the identified model captures the dynamics of interest very well. 
Table 4. Analytical results and estimation errors of blade in-plane modal parameters.

\begin{tabular}{rrr|rrr|rrr}
\hline \multicolumn{3}{c|}{ Frequencies } & \multicolumn{2}{c|}{ Damping factors } & \multicolumn{2}{c}{ Participation factors } \\
\hline \multirow{2}{*}{ Analytical } & \multicolumn{2}{c}{ Relative error } & Analytical & \multicolumn{2}{c}{ Relative error } & Analytical & \multicolumn{2}{c}{ Absolute error } \\
& PARX & POMA & PARX & POMA & POMA \\
\hline 0.4796 & 0.0015 & 0.0011 & 0.0367 & 0.0071 & 0.8773 & 0.0010 & -0.0009 & 0.0261 \\
0.6712 & 0.0010 & -0.0005 & 0.0262 & 0.0075 & 0.6569 & 0.0208 & -0.0038 & 0.0579 \\
0.8628 & 0.0008 & -0.0003 & 0.0204 & 0.0077 & 0.0325 & 0.9584 & 0.0074 & -0.1757 \\
1.0544 & 0.0007 & -0.0048 & 0.0167 & 0.0079 & 1.3739 & 0.0181 & -0.0011 & 0.0494 \\
1.2461 & 0.0006 & -0.0009 & 0.0141 & 0.0080 & 0.7958 & 0.0016 & -0.0015 & 0.0425 \\
\hline
\end{tabular}

Table 5. Analytical results and estimation errors of tower side-side modal parameters.

\begin{tabular}{rrr|rrr|rrr}
\hline \multicolumn{3}{c|}{ Frequencies } & \multicolumn{3}{c|}{ Damping factors } & \multicolumn{3}{c}{ Participation factors } \\
\hline \multirow{2}{*}{ Analytical } & \multicolumn{2}{c|}{ Relative error } & \multirow{2}{*}{ Analytical } & \multicolumn{2}{c}{ Relative error } & \multirow{2}{*}{ Analytical } & \multicolumn{2}{c}{ Absolute error } \\
& PARX & POMA & & & & & \\
PARX & POMA & & POMA \\
\hline 0.0374 & -0.0066 & -0.0276 & 0.1874 & -0.0887 & 0.8103 & 0.0000 & 0.0000 & 0.0081 \\
0.1550 & 0.0007 & 0.0014 & 0.0453 & -0.0953 & 0.7953 & 0.0000 & 0.0085 & 0.0196 \\
0.3466 & 0.0003 & 0.0009 & 0.0202 & -0.0950 & 0.0030 & 0.9990 & -0.0250 & -0.1375 \\
0.5383 & 0.0002 & 0.0006 & 0.0130 & -0.0949 & 0.7199 & 0.0000 & 0.0161 & 0.0640 \\
0.7299 & 0.0002 & -0.0022 & 0.0096 & -0.0948 & 2.8363 & 0.0000 & 0.0013 & 0.0468 \\
\hline
\end{tabular}

Table 4 reports the Floquet modal parameters, assumed as ground truth, as well as the errors obtained by the two methods considered here.

Looking at the results, it appears that both the PARX and POMA methods are able to capture the relevant dynamics related to the principal harmonics, as frequencies, damping, and participation factors are of good quality. In particular, damping and participation factors are slightly better estimated by PARX.

The estimation of the super-harmonic modal parameters deserves a special mention. The PARX method is able to provide a good matching for all modal parameters of all harmonics: frequencies and participation factors have negligible errors, whereas damping factors show an error lower than $1 \%$. On the other hand, the error of the POMA super-harmonic estimates is typically quite large especially for the damping factors, even though the principal harmonic is well captured.

This fact has mainly two possible explanations. First, the hypothesis of well-separated modes is here not fully satisfied, as the side band of the tower principal harmonic affects all super-harmonic peaks. The lower the rotor speed, the more pronounced this effect is, as the frequency separations among super-harmonics coincide with multiples of the rotor frequency. Second, but more importantly, according to the dynamics of a periodic system all harmonics belonging to a specific mode descend from a sole characteristic multiplier. Therefore, their frequencies and damping factors are strictly connected to each other. This relation is totally ignored by POMA (cf. Allen et al., 2011b), as it considers each peak in the frequency response as a stand-alone mode.

\subsubsection{Identification of other low-damped modes}

The tower side-side and blade in-plane whirling modes were excited by imposing different initial conditions for each blade edgewise angle and a suitable lateral displacement of the tower.

Figure 3 shows the harmonic power spectral density (HPSD) for the tower side-side displacement $y_{H}$, with frequency shifts from $-2 \Omega$ to $+2 \Omega$. Here again, the 0 -shift curve shows three distinct peaks: the tower side-side mode and the backward and forward in-plane whirling modes, respectively, at $0.34,0.68$ and $1.1 \mathrm{~Hz}$. Accordingly, the PARX complexity was set as $N_{a}=6, N_{b}=1, N_{F_{a}}=1$, and $N_{F_{b}}=$ 1. As for the previous case, the matching between predicted and simulated output, not reported here, is excellent. Comparisons among the exact and identified modal parameters are displayed in Table 5 through Table 7.

Figure 3 clearly shows that a good mode separation is not fully achieved here, as whirling super-harmonics interact with each other. This is not due to the specific wind turbine or condition considered here, as in fact any rotating blade system will always have the principal harmonics of its whirling modes separated by about $2 \Omega$. In addition, it also appears that the second super-harmonic of the tower mode at $0.73 \mathrm{~Hz}$ is very close to the second super-harmonic of the forward (FW) whirling mode at $0.71 \mathrm{~Hz}$; additionally, both harmonics are close to the backward (BW) whirling mode at $0.68 \mathrm{~Hz}$. For this reason, there are missing values in Table 5 through Table 7, wherever it was not possible to pick all peaks for all modes of interest using POMA. 
Table 6. Analytical results and estimation errors of in-plane backward whirling modal parameters.

\begin{tabular}{rrr|rrr|rrr}
\hline \multicolumn{3}{c|}{ Frequencies } & \multicolumn{3}{c|}{ Damping factors } & \multicolumn{3}{c}{ Participation factors } \\
\hline \multirow{2}{*}{ Analytical } & \multicolumn{2}{c|}{ Relative error } & \multirow{2}{*}{ Analytical } & \multicolumn{2}{c}{ Relative error } & \multirow{2}{*}{ Analytical } & \multicolumn{2}{c}{ Absolute error } \\
& PARX & POMA & & & & & \\
PARX & POMA & & POMA \\
\hline 0.3050 & -0.0135 & - & 0.0619 & -0.1675 & - & 0.0000 & 0.0007 & - \\
0.4964 & -0.0081 & -0.0096 & 0.0380 & -0.1720 & 5.3415 & 0.0000 & 0.0258 & 0.1234 \\
0.6880 & -0.0058 & -0.0040 & 0.0274 & -0.1739 & 0.0740 & 0.9889 & -0.0533 & -0.6323 \\
0.8796 & -0.0045 & 0.0002 & 0.0215 & -0.1750 & 0.7516 & 0.0000 & 0.0339 & 0.0315 \\
1.0712 & -0.0037 & 0.0056 & 0.0176 & -0.1757 & 0.3572 & 0.0000 & 0.0040 & 0.2431 \\
\hline
\end{tabular}

Table 7. Analytical results and estimation errors of in-plane forward whirling modal parameters.

\begin{tabular}{rrr|rrr|rrr}
\hline \multicolumn{3}{c|}{ Frequencies } & \multicolumn{3}{c|}{ Damping factors } & \multicolumn{3}{c}{ Participation factors } \\
\hline \multirow{2}{*}{ Analytical } & \multicolumn{2}{c|}{ Relative error } & \multirow{2}{*}{ Analytical } & \multicolumn{2}{c}{ Relative error } & \multirow{2}{*}{ Analytical } & \multicolumn{2}{c}{ Absolute error } \\
& PARX & POMA & & & & PORX & POMA \\
\hline 0.7108 & 0.0012 & - & 0.0281 & 0.0192 & - & 0.0000 & 0.0061 & - \\
0.9024 & 0.0010 & 0.0025 & 0.0222 & 0.0195 & 0.9598 & 0.0000 & 0.0411 & 0.0579 \\
1.0940 & 0.0008 & -0.0005 & 0.0183 & 0.0197 & 0.0000 & 0.9610 & -0.0168 & -0.3787 \\
1.2857 & 0.0007 & -0.0027 & 0.0156 & 0.0198 & 0.8850 & 0.0000 & 0.0084 & 0.0566 \\
1.4773 & 0.0006 & -0.0010 & 0.0135 & 0.0199 & 0.8131 & 0.0000 & 0.0000 & 0.0239 \\
\hline
\end{tabular}

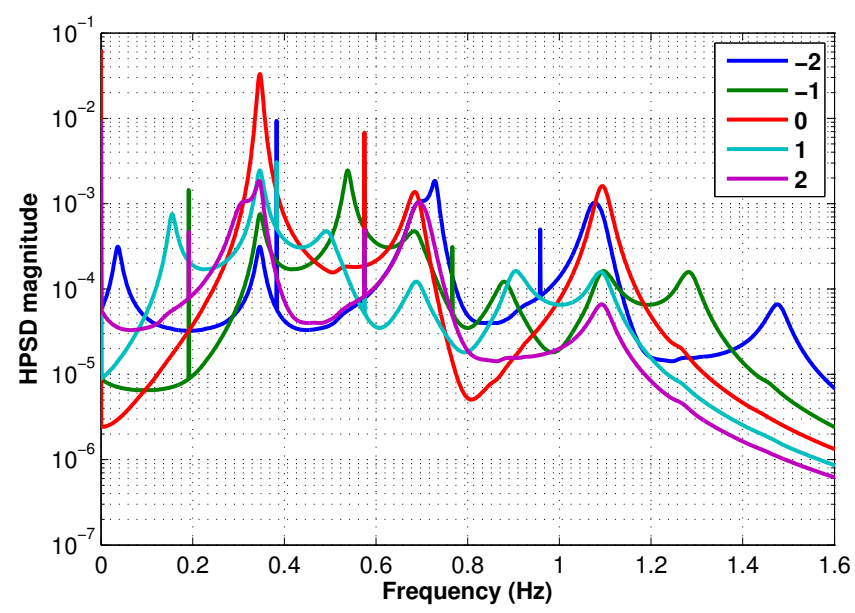

Figure 3. Harmonic power spectrum of the $y_{H}$ output of the wind turbine analytical model. Three modes are visible on the $n=0$ curve, along with the rotational frequency and its harmonics.

Considerations similar to ones previously made for the blade in-plane mode can also be stated here for these other three modes. Specifically, the frequency and damping factors of the principal harmonic of all modes are almost perfectly captured by both methods. The PARX method is the one that gives the most accurate results globally for both principal and super-harmonics: damping and participation factor estimates are characterized by small errors, while only the damping factors of the backward whirling mode have errors greater than $10 \%$. On the other hand, the POMA technique does not provide consistent results for the super-harmonic damping factors, which are characterized by large errors even when the damping factor of the principal harmonic is well captured. Moreover, the participation factors of the whirling modes exhibit non negligible errors for both principal and super-harmonics. This last issue is mainly due to the fact that, especially for the whirling case, the underlying hypothesis of well-separated modes is not completely fulfilled, as previously mentioned.

\section{PARMAX-based damping estimation using a high-fidelity multi-body model}

A detailed $6 \mathrm{MW}$ wind turbine high-fidelity multi-body model operating in a closed loop, implemented with the aeroservo-elastic simulator $\mathrm{Cp}$-Lambda (Bottasso and Croce, 2006-2016), was then used for a comparison of the POMA and the proposed PARMAX stability analysis techniques in a more sophisticated setting. Blades and tower are modeled with geometrically exact beam elements, discretized in space using the finite-element method, whereas the classical blade element momentum (BEM) theory is used to model the aerodynamics, with the usual inclusion of wake swirl, tip and hub losses, unsteady corrections, and dynamic stall. The total number of degrees of freedom in the resulting finite-element multi-body model is about 2500. A pitch-torque controller complements the aero-servo-elastic model. Wind histories compliant with IEC-61400 design guidelines were generated through TurbSim (Jonkman and Kilcher, 2012). The considered wind fields are characterized by a $5 \%$ turbulence 

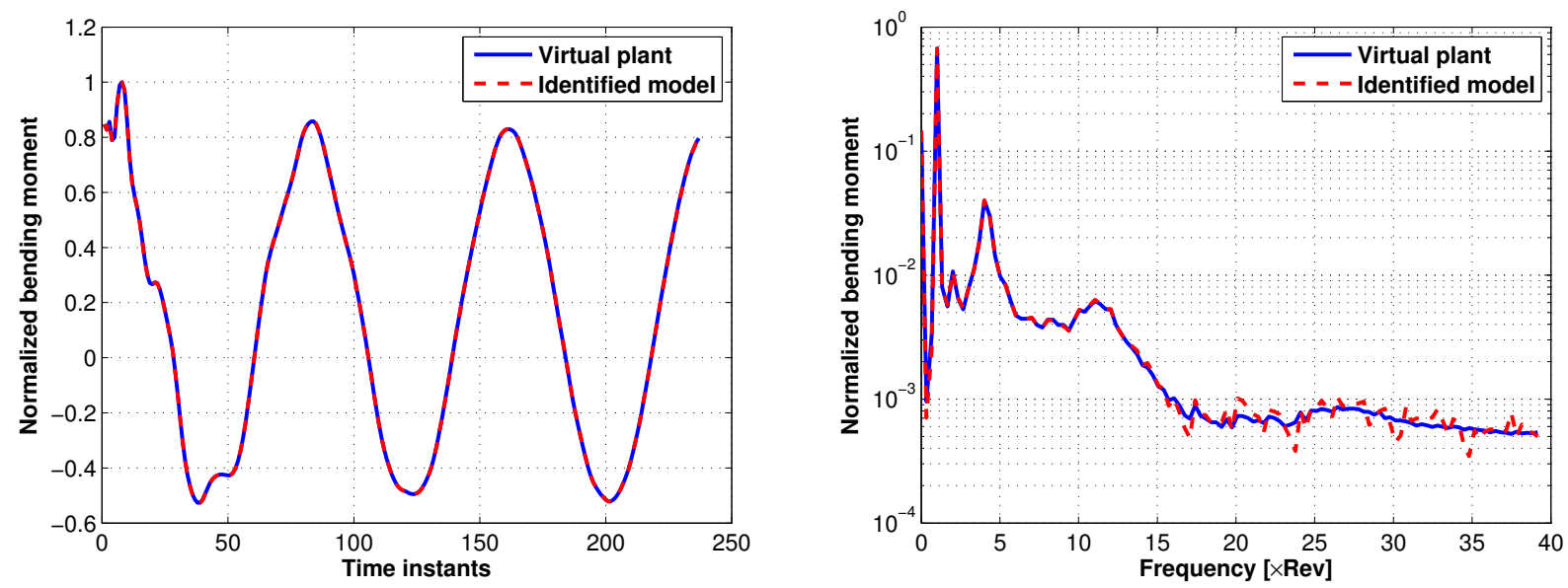

Figure 4. Comparison between measured (solid line) and predicted (dashed line) normalized blade root edgewise bending moment, in the time (left) and frequency (right) domains.
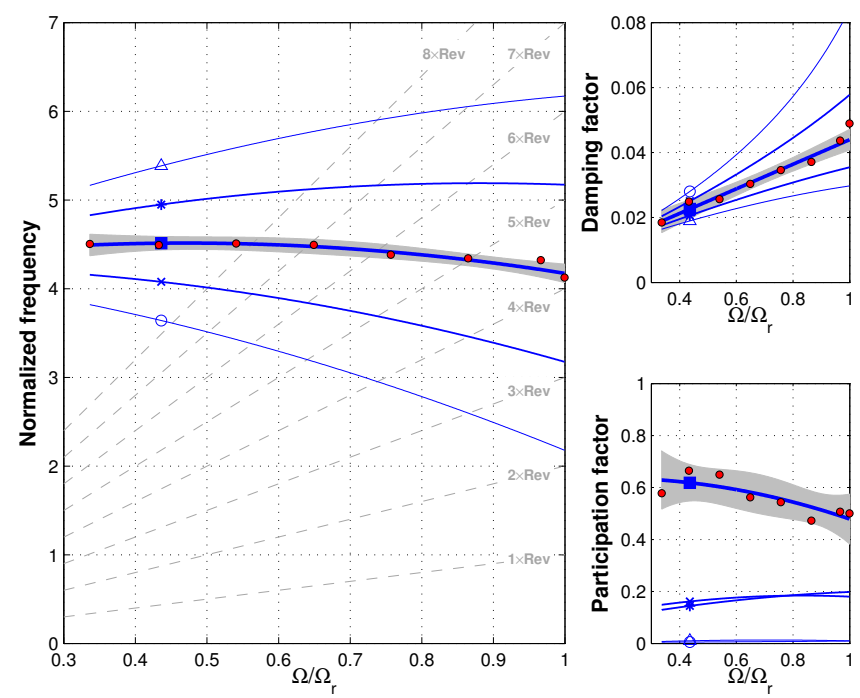

Figure 5. Periodic Campbell diagram of the first blade edgewise mode obtained from PARMAX identifications. The results of the single identifications along with the confidence level of the fitting curves are shown. Participation factors are computed in the rotating reference frame.

intensity and 10 min averaged wind speeds ranging from 3 to $10 \mathrm{~m} \mathrm{~s}^{-1}$, an upflow of $8^{\circ}$, and an atmospheric boundary layer power law exponent equal to 0.2 .

According to the PARMAX-based stability analysis, the system should be perturbed so as to induce a significant response of one or more modes of interest. Among the many possible ways of exciting a specific wind turbine mode, as, for example, the use of pitch and torque actuators (M. H. Hansen et al., 2006) or of eccentrical masses (Thomsen et al., 2000), impulsive forces were used in this work. Such forces could be realized in practice by pyrotechnic exciters. The rotor angular speed is averaged over

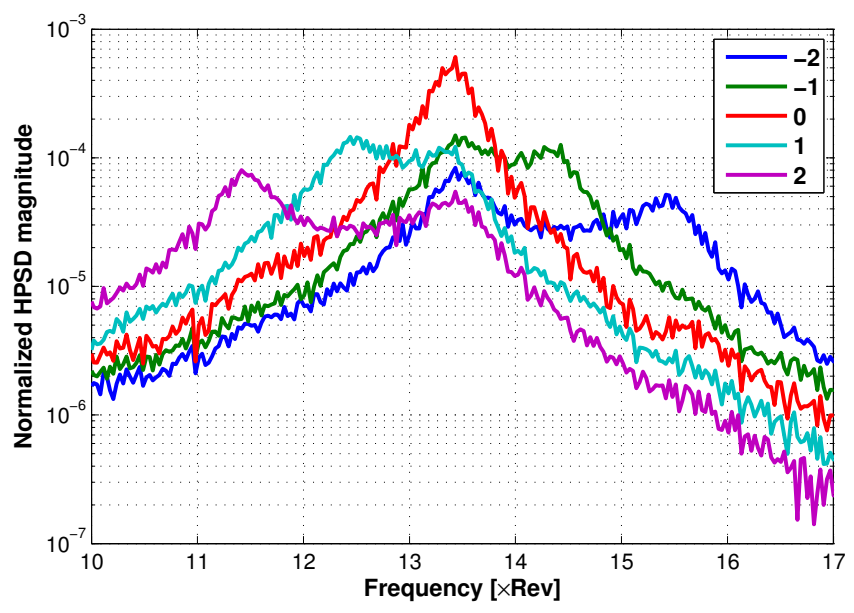

Figure 6. HPSD for the blade in-plane mode, obtained for a $3 \mathrm{~m} \mathrm{~s}^{-1}$ average wind speed.

the length of the recorded history and used to compute the system period. Afterwards, the signal is resampled in order to have an integer number of steps within a period.

The selection of the model complexity deserves special care. As the order of the AR part, $N_{a}$, is strictly related to the number of system modes, it can be estimated by looking at the number of principal-harmonic peaks present in the output PSD. This heuristic approach for the problem at hand turned out to be simple and effective and was preferred to more sophisticated criteria (Skjoldan and Bauchau, 2011; AvendañoValencia and Fassois, 2014). As described in Sect. 2.1, the input wind speed was considered as the sum of two contributions, a constant deterministic part and a turbulence-induced one. As long as the deterministic input is considered to be constant, one is allowed only to estimate an X part with or$\operatorname{der} N_{b}=1$. The MA-part order (noted as $N_{g}$ ) as well as the number of harmonics used to model the periodicity of the co- 

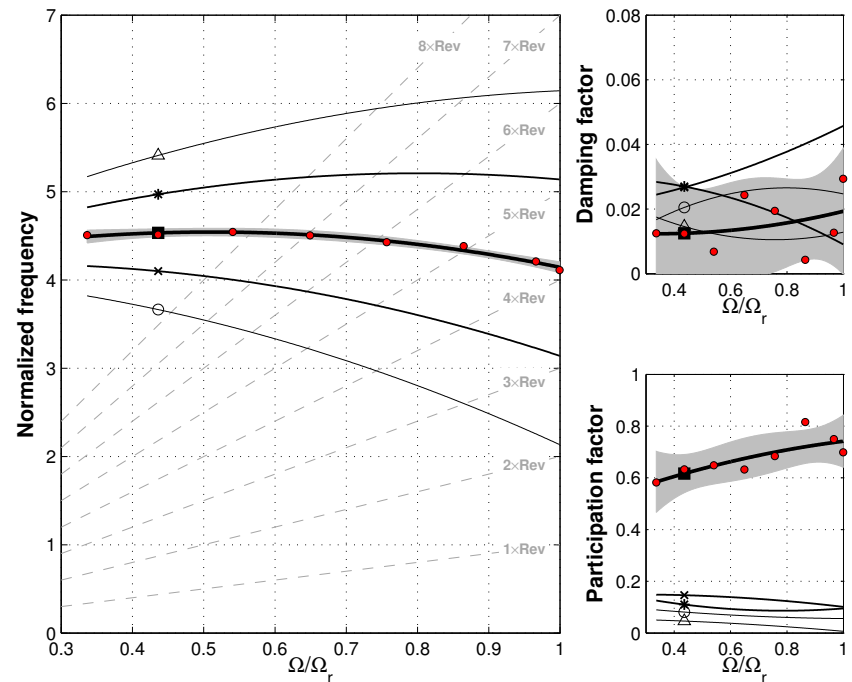

Figure 7. Periodic Campbell diagram of the first blade edgewise mode obtained from POMA identifications.

efficients (noted as $N_{F_{a}}, N_{F_{b}}$ and $N_{F_{g}}$ ) were set with a trial an error approach, until the achievement of satisfactory results.

After having performed the estimation for different wind conditions and therefore at different rotor speeds, the results of the analyses in terms of frequency, damping, and participation factors were fitted using low-order polynomials, computed by means of the robust bi-square algorithm (Kutner et al., 2005). The fitting process was applied only to the frequency and damping of the principal harmonic, indicated with the subscript $(\cdot)_{0}$. The corresponding characteristic exponent was then computed as

$\eta_{j_{0}}=-\omega_{j_{0}} \xi_{j_{0}}+\imath \omega_{j_{0}} \sqrt{1-\xi_{j}^{2}}$.

The super-harmonics were finally obtained by means of Eq. (A17). On the other hand, the participation factors of all super-harmonics were fitted with the same bi-square algorithm.

\subsection{Blade edgewise mode}

Two mainly edgewise doublets, applied at mid span and near the tip of the blade, were used to excite this mode. The PARMAX reduced-order model considered the following choice of parameters: $N_{a}=6, N_{F_{a}}=1, N_{b}=1, N_{F_{b}}=1, N_{g}=2$, and $N_{F_{g}}=0$. This setting allows for the modeling of three periodic modes.

The result of an identification executed at the rated rotor speed is shown in Fig. 4. The excellent superposition of the curves indicates a reduced-order PARMAX model of very good quality, capable of modeling the signal behavior despite the small nonlinearities and rotor speed variations characterizing the system that generated the data.

To draw the Campbell diagram, eight different identifications were made in order to cover the entire range of angular speeds of the machine. The results are shown in Fig. 5, where red dots indicate each specific identification, whereas lines refer to their quadratic fits. The gray bands are the $2 \sigma$ nonsimultaneous functional prediction bounds, and measure the confidence level of the fitting curves. From the gray bands one can infer that each frequency and damping factor identified at a specific rotor speed is coherent with the others, as all the estimates define a clear trend. On the other hand, a significant but acceptable uncertainty still characterizes the participation factors.

Similar analyses were conducted by Bottasso et al. (2014), where a different turbulence intensity (IEC level "B", instead of a uniform $5 \%$ over the whole wind speed range) was used, caeteris paribus. As the Campbell diagram is similar in both works, one may conclude that the PARMAX-based analysis does not appear to be significantly influenced by turbulence level.

Much longer portions of the time histories analyzed with PARMAX were then processed with the POMA method. In Fig. 6, the HPSD obtained for a wind field with a $3 \mathrm{~m} \mathrm{~s}^{-1}$ average speed is shown (note the similarities with Fig. 2). For this case the turbulence intensity was quite low, and the HPSD lines present well-defined peaks. However it was found that, for increasing wind speed, while the $n=0$ lines remain well defined, the quality of the peaks associated with the super-harmonics progressively degrades, making the estimation of damping (and, in some cases, also of frequency) increasingly more difficult.

The Campbell diagram obtained from POMA is displayed in Fig. 7. Comparing this figure with the PARMAX plot shows that frequencies are well identified, but the high dispersion of damping factors masks the expected trend. Several differences may also be seen between the plots with respect to the participation factors. While both approaches indicate that the principal harmonic is the most important in the response, they do, however, detect a markedly different behavior as a function of rotor speed. In addition, POMA overestimates the participation factors of the \pm 2 super-harmonics.

\subsection{Tower side-side mode}

The tower side-side mode was excited with a chirp-shaped force applied at the tower top. The frequency band of such signal was set in order to excite only that single mode. The tower base side-side moment was then recorded and used as output. As only the tower side-side peak is visible in the PSD of the response, then $N_{a}$ was set equal to 2 . The other coefficients were set as $N_{F_{a}}=1, N_{b}=1, N_{F_{b}}=1, N_{g}=2$, and $N_{F_{g}}=1$.

The agreement between the output predicted with the PARMAX reduced model and the measure, not shown here for the sake of brevity, is very good. The left plot of Fig. 8 shows the Campbell diagram obtained with the PARMAX approach. In this diagram the results of the identifications are approximated with straight lines. Looking at this plot, it 

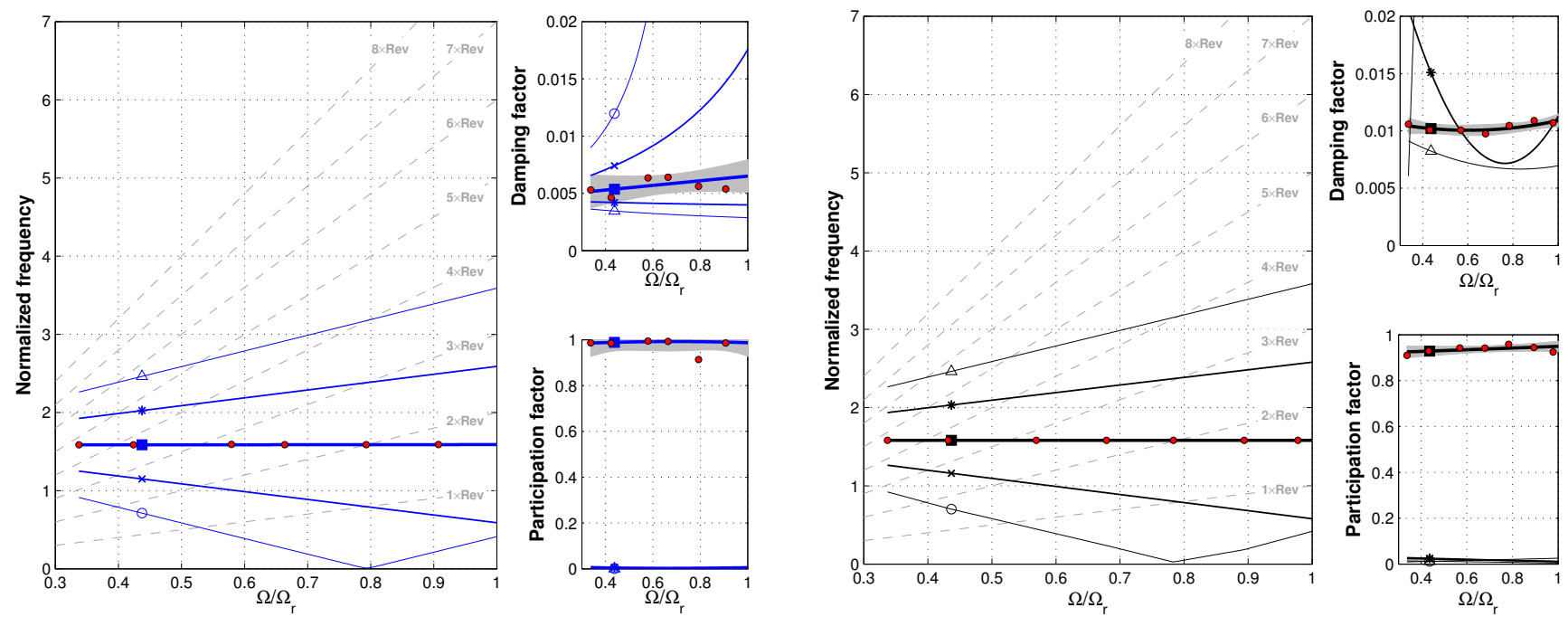

Figure 8. Periodic Campbell diagram for the tower side-side mode obtained from PARMAX (left) and POMA (right) identifications.

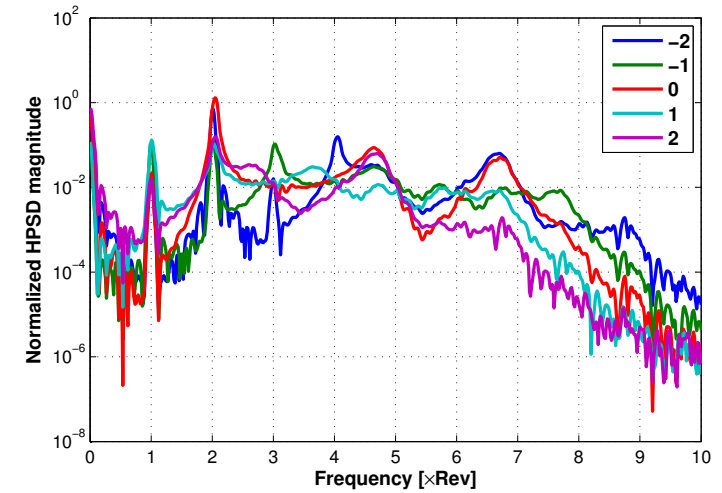

Figure 9. HPSD of the $M_{d}$ load, obtained for a $7 \mathrm{~m} \mathrm{~s}^{-1}$ average wind speed.

appears that at $0.8 \Omega_{r}$ the principal harmonic intersects the $2 \times$ Rev. For the PARMAX identification this is not particularly problematic, and in fact only the participation factor has been slightly underestimated. On the other hand, this poses a major problem for POMA. In fact, when the signal is frequency-shifted by $+2 \Omega$, its average value is transported over the principal peak, making it difficult to estimate the mode shape and the damping of the tower side-side mode.

The Campbell diagram obtained from POMA identifications is shown in the right-hand plot of Fig. 8. The plot clearly shows that the damping of the principal harmonic estimated with the half-power bandwidth is double the one estimated by PARMAX.

\subsection{Backward and forward whirling in-plane modes}

The backward and forward whirling in-plane modes were excited with a tower top side-side doublet, whose amplitude and duration were selected such that the input force spectrum is almost flat in the frequency range of interest. The threeblade root edgewise bending moments $M_{1}, M_{2}$, and $M_{3}$ were recorded, and the multi-blade coordinate transformation

$\left(\begin{array}{l}M_{0} \\ M_{d} \\ M_{q}\end{array}\right)=\frac{1}{3}\left[\begin{array}{ccc}1 & 1 & 1 \\ 2 \cos \left(\psi_{1}\right) & 2 \cos \left(\psi_{2}\right) & 2 \cos \left(\psi_{3}\right) \\ 2 \sin \left(\psi_{1}\right) & 2 \sin \left(\psi_{2}\right) & 2 \sin \left(\psi_{3}\right)\end{array}\right]\left(\begin{array}{l}M_{1} \\ M_{2} \\ M_{3}\end{array}\right)$

was used to yield the direct and quadrature moments, noted, respectively, as $M_{d}$ and $M_{q}$. The spectra of $M_{d}$, displayed in Fig. 9, show well-defined peaks.

The PARMAX reduced model was set with the following choice of parameters: $N_{a}=8, N_{F_{a}}=1, N_{b}=1, N_{F_{b}}=$ $1, N_{g}=2$, and $N_{F_{g}}=1$. Both the backward and forward whirling in-plane modes, as well as the side-side tower mode, were nicely visible in the frequency plot of the perturbed time histories. Thus, for each wind speed, only one reduced model capable of representing the behavior of all these three modes was identified.

Figures 10 and 11 show on the left the periodic Campbell diagram obtained using the PARMAX approach and on the right the one computed with POMA, respectively, for the backward and the forward whirling in-plane modes. It should be noted that both approaches provide the same results in terms of frequencies. The overall trend of the principalharmonic damping factors as functions of the rotor speed is similarly captured. In particular, the PARMAX results are characterized by a lower uncertainty for the backward mode and a higher uncertainty for the forward one. The rising of the damping factors with the angular speed, for these two modes, has been observed also in Skjoldan and Hansen (2013), although for an isotropic condition.

Once again, the damping of the super-harmonics obtained with the POMA technique are not well estimated, as already noted in Sect. 4. Moreover, the participation factors of the 

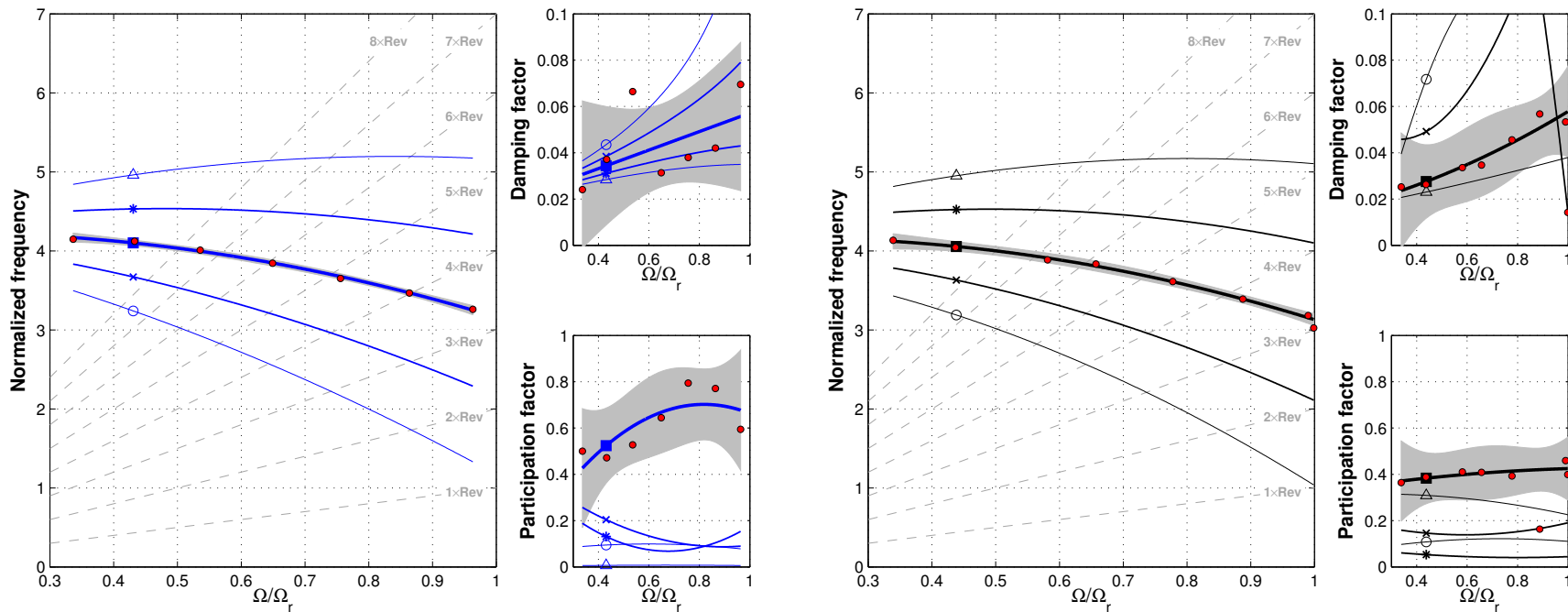

Figure 10. Periodic Campbell diagram of the backward whirling in-plane mode obtained from PARMAX (left) and POMA (right) identifications.
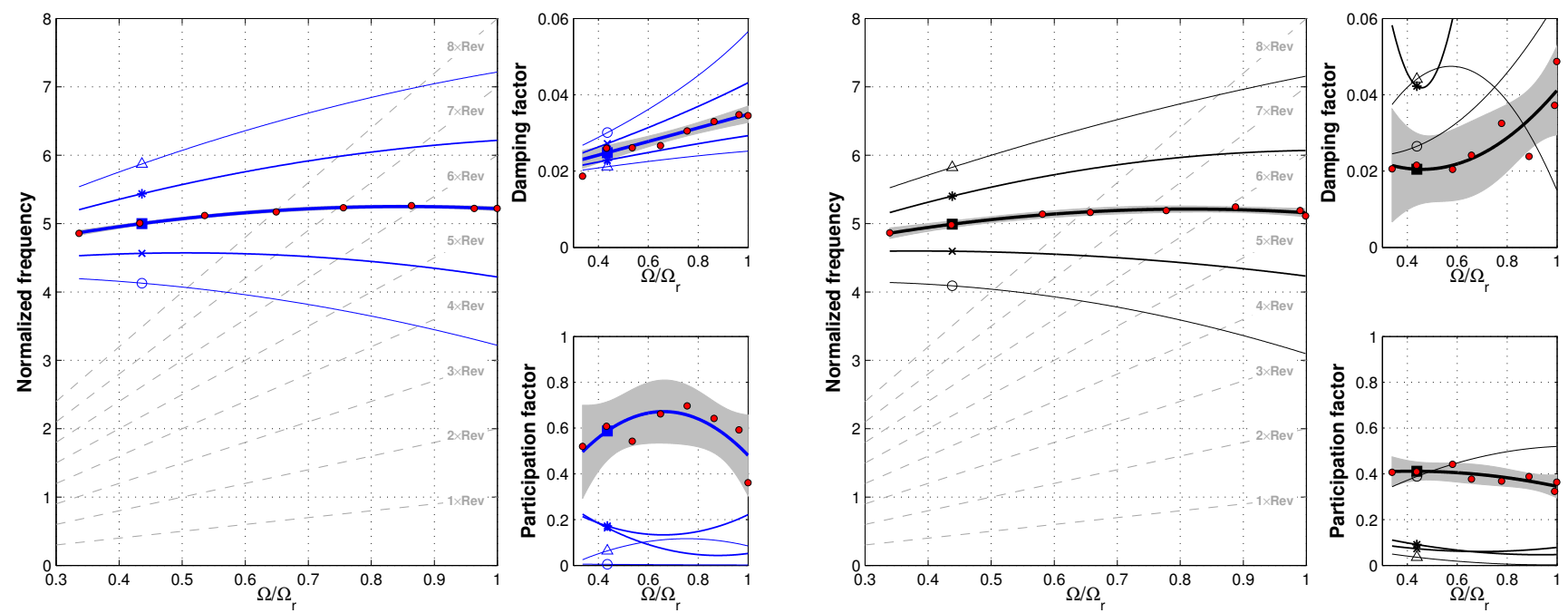

Figure 11. Periodic Campbell diagram of the forward whirling in-plane mode obtained from PARMAX (left) and POMA (right) identifications.

\pm 2 super-harmonics are typically too high: for example, in the right-hand plot of Fig. 11, one can see that the participation of super-harmonic +2 of the forward whirling mode is higher than that of the principal one. This strongly overestimated participation is due to the nearly $2 \Omega$ spacing of the whirling modes, which causes their super-harmonics to nearly overlap.

\section{Conclusions}

In this paper we have considered a model-independent periodic stability analysis capable of handling turbulent disturbances. The approach is based on the identification of a PAR-
MAX reduced model from a transient response of the machine. The full Floquet theory is then applied to the reduced model, yielding all modal quantities of interest. As only time series of measurements are necessary, the method appears to be suitable for the application to real wind turbines operating in the field.

In order to assess the validity of the proposed method, the well-known POMA was implemented and used for comparison. Tests were performed first with the help of a wind turbine analytical model, whose exact solution can be obtained by the theory of Floquet, and then with a high-fidelity wind turbine multi-body model operating in turbulent wind conditions. 
Based on the results obtained in this study, one may draw the following considerations.

- Both methods are able to characterize the relevant behavior of the wind turbine in turbulent wind conditions. However, the results provided by the proposed PARMAX analysis are in general more accurate than those given by the POMA technique, especially if one looks not only at the principal harmonics but also at the superharmonics.

- Often the underlying hypotheses of POMA are not exactly fulfilled, and this leads to inaccuracies especially in terms of damping and participation factors. These effects are more visible for the whirling modes, as they are separated by about $2 \Omega$, which means that there will always be a perfect overlap between the super-harmonics of these two modes at some angular velocity. The PARMAX analysis is less prone to such problems.

- A major advantage of PARMAX over POMA is that it requires shorter time histories. This is important in turbulent conditions, where the rotor speed is hardly constant (which, on the other hand, is a fundamental hypothesis of both methods).
The development of the present SISO PARMAX approach suggests a number of extensions, which are currently under investigation.

- The use of multiple outputs in a multiple-input multipleoutput (MIMO) PARMAX framework could improve the quality of the results.

- Due to the stochastic nature of turbulence, a multihistory PARMAX applied to different realizations of the same experiment could provide more robust modal results, along with the associated variances.

- The peak-picking method is rather simple, and it is unable to exploit all the informational content available in the HPSD, especially in the presence of noisy peaks. Fitting algorithms have been preliminarily explored (see Allen et al., 2011a), but their application to the multiple output case has not yet been attempted. 
Appendix A: Review of linear time periodic systems

\section{A1 Floquet theory in continuous time}

A generic SISO LTP system in continuous time can be written in state space form as

$\dot{\boldsymbol{x}}=\mathbf{A}(t) \boldsymbol{x}+\mathbf{B}(t) u$,

$y=\mathbf{C}(t) \boldsymbol{x}+\mathbf{D}(t) u$

where $t$ is time and $\boldsymbol{x}, u$, and $y$ the state, input, and output vectors, respectively, while $\mathbf{A}(t), \mathbf{B}(t), \mathbf{C}(t)$, and $\mathbf{D}(t)$ are periodic system matrices such that

$\mathbf{A}(t+T)=\mathbf{A}(t), \quad \mathbf{B}(t+T)=\mathbf{B}(t)$,

$\mathbf{C}(t+T)=\mathbf{C}(t), \quad \mathbf{D}(t+T)=\mathbf{D}(t)$,

for any $t$. The smallest $T$ satisfying Eq. (A2) is defined as the system period. Scalar $u$ can be any of the wind turbine control inputs (i.e., blade pitch angles, electrical torque, possibly the yaw angle) as well as exogenous inputs related to the wind states (e.g., wind speed, vertical or lateral shears, cross-flow).

To study the stability of Eq. (A1a), its autonomous version is considered together with the associated initial conditions:

$\dot{\boldsymbol{x}}=\mathbf{A}(t) \boldsymbol{x}, \quad \boldsymbol{x}(0)=\boldsymbol{x}_{0}$.

The state transition matrix $\boldsymbol{\Phi}(t, \tau)$ maps the state at time $\tau$, $\boldsymbol{x}(\tau)$, onto the state at time $t, \boldsymbol{x}(t)$,

$\boldsymbol{x}(t)=\boldsymbol{\Phi}(t, \tau) \boldsymbol{x}(\tau)$,

and it obeys a similar equation with its associated initial conditions

$\dot{\boldsymbol{\Phi}}(t, \tau)=\mathbf{A}(t) \boldsymbol{\Phi}(t, \tau), \quad \boldsymbol{\Phi}(\tau, \tau)=\mathbf{I}$,

where $\mathbf{I}$ is the identity matrix. It can be shown that in the continuous-time case the transition matrix is always invertible (Bittanti and Colaneri, 2009).

An important role in the stability analysis of periodic systems is played by the state transition matrix over one period $\boldsymbol{\Psi}(\tau)=\boldsymbol{\Phi}(\tau+T, \tau)$, termed monodromy matrix. By definition, the monodromy matrix relates two states separated by a period; consequently, a generic state that is sampled at every period, noted as $\widetilde{\boldsymbol{x}}_{\tau}(k)=\boldsymbol{x}(\tau+k T)$, obeys the following linear-invariant discrete-time equation

$\widetilde{\boldsymbol{x}}_{\tau}(k+1)=\boldsymbol{\Psi}(\tau) \widetilde{\boldsymbol{x}}_{\tau}(k)$

The system is asymptotically stable if all the eigenvalues of the monodromy matrix, characteristic multipliers and noted as $\theta_{j}$, belong to the open unit disk in the complex plane. It can be shown that the eigenvalues of the monodromy matrix and their multiplicity are time-invariant even if the monodromy matrix is periodic (Bittanti and Colaneri, 2009). For this reason, one can ignore the time lag $\tau$ when referring to the characteristic multipliers. The eigenvalues $\theta_{j}$ and associated eigenvectors $\boldsymbol{s}_{j}$ are obtained by the spectral factorization of the monodromy matrix, i.e.,

$\boldsymbol{\Psi}(\tau)=\operatorname{Sdiag}\left(\theta_{j}\right) \mathbf{S}^{-1}$,

with $\mathbf{S}=\left[\ldots, \boldsymbol{s}_{j}, \ldots\right]$.

In order to determine the frequency content of a periodic system, it is necessary to introduce the so-called FloquetLyapunov transformation. The Floquet-Lyapunov problem is the one of finding a bounded, periodic, and invertible state space transformation $\boldsymbol{z}(t)=\mathbf{Q}(t) \boldsymbol{x}(t)$ such that the resulting governing equation

$\dot{z}=\mathbf{R} z$

is time-invariant, i.e., the Floquet factor matrix $\mathbf{R}$ is constant. Since $\mathbf{R}=\mathbf{Q}(t) \mathbf{A}(t) \mathbf{Q}^{-1}(t)+\dot{\mathbf{Q}}(t) \mathbf{Q}^{-1}(t)$, the periodic transformation $\mathbf{Q}(t)$ must obey the following matrix differential equation

$\dot{\mathbf{Q}}(t)=\mathbf{R} \mathbf{Q}(t)-\mathbf{Q}(t) \mathbf{A}(t)$,

whose solution is

$\mathbf{Q}(t)=e^{\mathbf{R}(t-\tau)} \mathbf{Q}(\tau) \boldsymbol{\Phi}^{-1}(t, \tau)$.

Exploiting the periodicity condition $\mathbf{Q}(\tau+T)=\mathbf{Q}(\tau)$, one gets the relationship between monodromy matrix and Floquet factor, which writes

$\boldsymbol{\Psi}(\tau)=\mathbf{Q}(\tau)^{-1} e^{\mathbf{R} T} \mathbf{Q}(\tau)$.

The eigenvalues of the Floquet factor, called characteristic exponents and noted as $\eta_{j}$, are computed by the spectral factorization of $\mathbf{R}$ :

$\mathbf{R}=\boldsymbol{V} \operatorname{diag}\left(\eta_{j}\right) \mathbf{V}^{-1}$,

with $\mathbf{V}=\left[\ldots, \boldsymbol{v}_{j}, \ldots\right]$. Inserting Eqs. (A7) and (A12) into Eq. (A11), the following result is derived

$\operatorname{diag}\left(\theta_{j}\right)=\mathbf{S}^{-1} \mathbf{Q}(\tau)^{-1} \mathbf{V} \operatorname{diag}\left(e^{\eta_{j} T}\right) \mathbf{V}^{-1} \mathbf{Q}(\tau) \mathbf{S}$,

which shows that $\mathbf{V}=\mathbf{Q}(\tau) \mathbf{S}$ and, more importantly, that characteristic multipliers and characteristic exponents are related as

$\theta_{j}=e^{\eta_{j} T}$.

Note that there is an infinite number of Floquet factors, and therefore an infinite number of Floquet-Lyapunov transformations. In fact, one can choose any invertible initial condition $\mathbf{Q}(\tau)$. In addition, computing characteristic exponents from multipliers by inverting Eq. (A14) leads to a multiplicity of solutions, as in fact

$\eta_{j}=\frac{1}{T} \ln \left(\theta_{j}\right)=\frac{1}{T}\left(\ln \left|\theta_{j}\right|+\imath\left(\angle\left(\theta_{j}\right)+2 \ell \pi\right)\right)$, 
where $\ell \in \mathbb{Z}$ is an arbitrary integer. This indeterminacy, however, does not affect the real frequency content of the response, since the transition matrix is uniquely defined. This aspect of the problem will be further analyzed later on in these notes.

Given $\mathbf{Q}(\tau)$ and $\mathbf{R}$, the transition matrix is readily obtained from Eq. (A10) as

$\boldsymbol{\Phi}(t, \tau)=\mathbf{P}(t) e^{\mathbf{R}(t-\tau)} \mathbf{P}(\tau)^{-1}$,

where the periodic matrix $\mathbf{P}(t)=\mathbf{Q}(t)^{-1}$ is termed periodic eigenvector.

Consider now, for each mode, one of the infinite solutions of Eq. (A15), for example, the one with $\ell=0$, noted as $\hat{\eta}_{j}$. Introducing $\Omega=2 \pi / T$, any other characteristic exponent $\eta_{j}$ could be computed from $\hat{\eta}_{j}$ as

$\eta_{j}=\hat{\eta}_{j}+\ln \Omega, \quad n \in \mathbb{Z}$.

Inserting Eq. (A12) into Eq. (A16), one can express the state transition matrix as the following modal sum:

$\boldsymbol{\Phi}(t, \tau)=\sum_{j=1}^{N_{\mathrm{S}}} \boldsymbol{Z}_{j}(t, \tau) e^{\hat{\eta}_{j}(t-\tau)}$,

where $\boldsymbol{Z}_{j}(t, \tau)=\mathbf{P}(t) \mathbf{V I} \mathbf{I}_{j j} \mathbf{V}^{-1} \mathbf{Q}(\tau)$, while $\mathbf{I}_{j j}$ is a matrix with the sole element $(j, j)$ equal to 1 and all others equal to 0 . Because of the particular definition of $\mathbf{I}_{j j}$, matrix $\boldsymbol{Z}_{j}(t, \tau)$ is of unitary rank $\forall(t, \tau)$, and it is also equal to $\boldsymbol{\psi}_{j}(t) \boldsymbol{L}_{j}(\tau)^{T}$, where

$$
\begin{aligned}
\boldsymbol{\psi}_{j}(t) & =\operatorname{col}_{j}(\boldsymbol{\Xi}(t)), \\
\boldsymbol{L}_{j}(\tau)^{T} & =\operatorname{row}_{j}\left(\boldsymbol{\Xi}^{-1}(\tau)\right),
\end{aligned}
$$

with $\boldsymbol{\Xi}(t)=\mathbf{P}(t) \mathbf{V}$. Equation (A18) can be now reformulated as

$$
\boldsymbol{\Phi}(t, \tau)=\sum_{j=1}^{N_{\mathrm{s}}} \boldsymbol{\psi}_{j}(t) \boldsymbol{L}_{j}(\tau)^{T} e^{\hat{\eta}_{j}(t-\tau)}
$$

Exploiting the periodicity of $\boldsymbol{\psi}_{j}(t)$, Eq. (A20) becomes

$\boldsymbol{\Phi}(t, \tau)=\sum_{j=1}^{N_{\mathrm{S}}} \sum_{n=-\infty}^{+\infty} \boldsymbol{\psi}_{j_{n}} \boldsymbol{L}_{j}(\tau)^{T} e^{\left(\hat{\eta}_{j}+\imath n \Omega\right)(t-\tau)} e^{\imath n \Omega \tau}$

where $\psi_{j_{n}}$ is the amplitudes of the harmonics of the Fourier expansion of $\boldsymbol{\psi}_{j}(t)$. This expression of the state transition matrix can also be found in Skjoldan and Hansen (2009), Allen et al. (2011b), and Bottasso and Cacciola (2015).

From Eq. (A21) it appears that, for each mode, an infinite number of exponents (playing the role of eigenvalues of the LTI system) participates in the response of the system. Furthermore, a single frequency is not sufficient for completely characterizing that mode. All exponents have imaginary parts that differ by integer multiples of $\Omega$ and have the same real part; thus, all exponents of a given mode are either stable or unstable. This fact is not surprising, as the stability of the system is just determined by the characteristic multipliers, which are uniquely defined.

For the LTP system, the exponents $\hat{\eta}_{j}+\imath n \Omega$ play the role of the eigenvalues of the LTI case, as they yield the frequencies $\omega_{j_{n}}=\left|\hat{\eta}_{j}+\imath n \Omega\right|$ and damping factors $\xi_{j_{n}}=$ $-\operatorname{Re}\left(\hat{\eta}_{j}\right) / \omega_{j_{n}}$ of each mode. To describe this situation, this infinite multiplicity of frequencies is termed a fan of modes (cf. Bottasso and Cacciola, 2015). Each harmonic in a fan contributes to the overall response according to its associated "modal shape" $\psi_{j_{n}}$. The relative contribution of the $n$th harmonic to the $j$ th mode is measured through its participation factor, defined as

$\phi_{j_{n}}=\frac{\left\|\psi_{j_{n}}\right\|}{\sum_{n}\left\|\psi_{j_{n}}\right\|}$.

The triads $\left\{\omega_{j_{n}}, \xi_{j_{n}}, \phi_{j_{n}}\right\}$ describe completely the behavior of a periodic mode. The participation factors can be defined also as functions of the Frobenius norm of the harmonics of $\boldsymbol{Z}_{j}(t, \tau), \boldsymbol{Z}_{j_{n}}=\boldsymbol{\psi}_{j_{n}} \boldsymbol{L}_{j}(\tau)^{T}$, as shown in Bottasso and Cacciola (2015):

$\phi_{j_{n}}=\frac{\left\|\boldsymbol{Z}_{j_{n}}\right\|_{\mathrm{F}}}{\sum_{n}\left\|\boldsymbol{Z}_{j_{n}}\right\|_{\mathrm{F}}}$.

The two definitions are exactly equivalent as, in this specific case, $\left\|\boldsymbol{Z}_{j_{n}}\right\|_{\mathrm{F}}=\left\|\boldsymbol{\psi}_{j_{n}}\right\|\left\|\boldsymbol{L}_{j}(\tau)\right\|$ and $\boldsymbol{L}_{j}(\tau)$ stays the same for all harmonics. ${ }^{1}$

The apparent indeterminacy in the computation of the imaginary part of the logarithm of the characteristic multipliers in Eq. (A15) is then understood. In fact, all the exponents that satisfy Eq. (A14) are present in the response of the system, as it can be seen from Eq. (A21). Since the transition matrix is uniquely defined, any choice of the integer $\ell$ in Eq. (A15) would act as a shift in the frequency content of $\boldsymbol{Z}_{j}$, such that all triads $\left\{\omega_{j_{n}}, \xi_{j_{n}}, \phi_{j_{n}}\right\}$ remain exactly the same, as first observed by Borri (1986) and later discussed by Peters et al. (2011).

Often, although not always, the harmonic with the highest participation is very similar in terms of frequency and damping to the one that would result from the invariant analysis of periodic systems based on the Coleman transformation (Coleman and Feingold, 1958; Hansen, 2004). As suggested by Bottasso and Cacciola (2015), such a harmonic may be called the principal one, while the others may be termed super-harmonics. Furthermore, any one of these harmonics could resonate with external excitations.

\footnotetext{
${ }^{1}$ Given two-column vectors $\quad \boldsymbol{v}=\left(\ldots, v_{i}, \ldots\right)^{T}$ and $\boldsymbol{w}=\left(\ldots, w_{j}, \ldots\right)^{T}$, the square of the Frobenius norm of the product $\boldsymbol{v} \boldsymbol{w}^{T}$ can be expressed as $\left\|\boldsymbol{v} \boldsymbol{w}^{T}\right\|_{\mathrm{F}}^{2}=\sum_{i} \sum_{j}\left(v_{i} w_{j}\right)^{2}=$ $\sum_{i} v_{i}^{2} \sum_{j} w_{j}^{2}=\|\boldsymbol{v}\|^{2}\|\boldsymbol{w}\|^{2}$.
} 
In order to understand how each harmonic appears in a specific output of the system, the output-specific participation factor can be defined. To this end, consider an output of the autonomous system Eq. (A3),

$y(t)=\mathbf{C}(t) \boldsymbol{\Phi}(t, \tau) \boldsymbol{x}(\tau)=\boldsymbol{\Phi}_{y}(t, \tau) \boldsymbol{x}(\tau)$.

Inserting Eq. (A20) into Eq. (A24) the following is derived:

$\boldsymbol{\Phi}_{y}(t, \tau)=\sum_{j=1}^{N_{\mathrm{S}}} \mathbf{C}(t) \boldsymbol{\psi}_{j}(t) \boldsymbol{L}_{j}(\tau)^{T} e^{\hat{\eta}_{j}(t-\tau)}$

Exploiting now the periodicity of the product $\mathbf{C}(t) \psi_{j}(t)$, Eq. (A25) can be rearranged as

$\boldsymbol{\Phi}_{y}(t, \tau)=\sum_{j=1}^{N_{\mathrm{S}}} \sum_{n=-\infty}^{+\infty} c_{j_{n}} e^{\left(\left(\hat{\eta}_{j}+\imath n \Omega\right)(t-\tau)\right)} \boldsymbol{L}_{j}(\tau)^{T} e^{\imath n \Omega \tau}$,

where $c_{j_{n}}$ is the harmonics of the Fourier expansion of $\mathbf{C}(t) \psi_{j}(t)$. The output-specific participation factor can finally be defined as

$\phi_{j_{n}}^{y}=\frac{\left|c_{j_{n}}\right|}{\sum_{n}\left|c_{j_{n}}\right|}$.

A2 The harmonic transfer function and the harmonic frequency response function

The forced response of system Eq. (A1), called $y_{\mathrm{F}}(t)$, can be computed as

$$
\begin{aligned}
& y_{\mathrm{F}}(t)=\int_{0}^{t} h(t, \sigma) u(\sigma) \mathrm{d} \sigma= \\
& \int_{0}^{t}(\mathbf{C}(t) \boldsymbol{\Phi}(t, \sigma) \mathbf{B}(\sigma)+D(\sigma) \delta(t-\sigma)) u(\sigma) \mathrm{d} \sigma,
\end{aligned}
$$

where

$$
h(t, \tau)=\mathbf{C}(t) \boldsymbol{\Phi}(t, \tau) \mathbf{B}(\tau)+\mathbf{D}(\tau) \delta(t-\tau)
$$

is the impulse response. From Eq. (A28), it appears that the periodicity of $\mathbf{C}(t), \mathbf{B}(t)$, and $\boldsymbol{\Phi}(t, \tau)$ results in an inputoutput behavior of an LTP system that is far from being describable as an LTI-like one. In particular, it can be shown that an LTP system subjected to an input at a given frequency may respond at an infinite number of frequencies, which in addition to the input frequency itself include also the integer multiples of the system frequency (Bittanti and Colaneri, 2009; Wereley, 1991). This is also the reason why any output of a wind turbine subjected to a constant-in-time wind (i.e., at the zero frequency) is characterized by frequencies at the multiples of the rotor speed (i.e., $0 \times \operatorname{Rev}, 1 \times \operatorname{Rev}, 2 \times \operatorname{Rev}$, ...).
In the frequency domain, the input-output relation can be expressed by means of the HTF (cf. Bittanti and Colaneri, 2009; Wereley, 1991), which can be interpreted as the extension to periodic systems of the standard time-invariant transfer function. To this end, the so-called exponentially modulated periodic (EMP) signal is defined as

$v(t)=\sum_{k \in \mathbb{Z}} v_{k} e^{(s+i k \Omega) t}$,

where $s \in \mathbb{C}$. According to definition Eq. (A30), any $v_{k}$ can be also viewed as the Laplace transformation of $v(t)$ evaluated at $s+\imath k \Omega$ as

$v_{k}(s)=\int_{-\infty}^{\infty} v(t) e^{-(s+l k \Omega) t} \mathrm{~d} t$

It can be shown that a periodic system subjected to an EMP admits an EMP regime (Bittanti and Colaneri, 2009) and that in such a regime its states are EMP signals. In order to exploit this property, one has first to define two doubly infinitedimensional vectors containing, respectively, the EMP harmonics $u(t)$ and $y(t)$, as

$\mathcal{Y}(s)=\left(\begin{array}{lllll}\cdots & y_{-1}(s) & y_{0}(s) & y_{1}(s) & \cdots\end{array}\right)^{T}$,
$\mathcal{U}(s)=\left(\begin{array}{lllll}\cdots & u_{-1}(s) & u_{0}(s) & u_{1}(s) & \cdots\end{array}\right)^{T}$.

Next, the doubly-infinite Toeplitz matrices $\mathcal{A}, \mathcal{B}, \mathcal{C}$, and $\mathcal{D}$, containing the Fourier expansions $\mathbf{A}_{k}, \mathbf{B}_{k}, \mathbf{C}_{k}$, and $\mathbf{D}_{k}$ of the corresponding system matrices, are defined as

$\mathcal{A}=\left[\begin{array}{ccccc}\ddots & \vdots & \vdots & \vdots & . \cdot \\ \cdots & \mathbf{A}_{0} & \mathbf{A}_{-1} & \mathbf{A}_{-2} & \cdots \\ \cdots & \mathbf{A}_{1} & \mathbf{A}_{0} & \mathbf{A}_{-1} & \cdots \\ \cdots & \mathbf{A}_{2} & \mathbf{A}_{1} & \mathbf{A}_{0} & \cdots \\ \cdot & \vdots & \vdots & \vdots & \ddots\end{array}\right]$

and similarly for the $\mathcal{B}, \mathcal{C}$, and $\mathcal{D}$ matrices. Finally, by inserting the EMP expansions of $y$ and $u$ and the Fourier expansions of the system matrices into Eq. (A1), summing up all terms at the same frequency, the input-output relationship is derived as

$\mathcal{Y}(s)=\mathcal{G}(s) \mathcal{U}(s)$

where the HTF is defined as

$\mathcal{G}(s)=\mathcal{C}(s \mathcal{I}-(\mathcal{A}-\mathcal{N}))^{-1} \mathcal{B}+\mathcal{D}$

with $\mathcal{N}=\operatorname{blkdiag}\{l k \Omega \mathbf{I}, k \in \mathbb{Z}\}$ and $\mathcal{I}$ and $\mathbf{I}$ being identity matrices of suitable dimensions

The HTF can also be represented by means of the impulse response of the system (Bittanti and Colaneri, 2009). From Eq. (A29), it is easily verified that function $h(t, t-r)$ for a 
fixed time lag $r$ is periodic and, consequently, that it can be expanded in a Fourier series as

$h(t, \tau)=\sum_{k=-\infty}^{\infty} h_{k}(t-\tau) e^{\imath k \Omega t}$

The output equation can then be written according to the following convolution:

$y(t)=\sum_{k=-\infty}^{\infty} \int_{0}^{t} h_{k}(t-\tau) e^{\imath k \Omega(t-\tau)} u(\tau) e^{\imath k \Omega \tau} \mathrm{d} \tau$,

which leads to the input-output relation in the Laplace domain:

$Y(s)=\sum_{k=-\infty}^{\infty} H_{k}(s-\imath k \Omega) U(s-\imath k \omega)$,

where $Y(s), U(s)$, and $H_{k}(s)$ are, respectively, the Laplace transforms of $y, u$, and $h_{k}$. Equation (A38) can be evaluated for each element of the EMP output signal $\mathcal{Y}$ by substituting the complex number $s$ with the exponentially modulated periodic one $s+\imath k \Omega$ with $k \in \mathbb{Z}$, leading to the following relationship:

$Y(s+\imath k \Omega)=\sum_{n=-\infty} H_{k-n}(s+\imath n \Omega) U(s+\imath n \Omega)$.

Consequently, since $Y(s+\imath k \Omega)=y_{k}(s)$ and $U(s+\imath k \Omega)=$ $u_{k}(s)$ because of Eq. (A31), the HTF can be written as

$$
\begin{aligned}
& \mathcal{G}(s)= \\
& {\left[\begin{array}{ccccc}
\ddots & \vdots & \vdots & \vdots & . \cdot \\
\cdots & H_{0}(s-\imath \Omega) & H_{-1}(s) & H_{-2}(s+\imath \Omega) & \cdots \\
\cdots & H_{1}(s-\imath \Omega) & H_{0}(s) & H_{-1}(s+\imath \Omega) & \cdots \\
\cdots & H_{2}(s-\imath \Omega) & H_{1}(s) & H_{0}(s+\imath \Omega) & \ldots \\
. & \vdots & \vdots & \vdots & \ddots
\end{array}\right] .}
\end{aligned}
$$

Inserting Eq. (A21) into Eq. (A29), one can derive the following expression

$$
\begin{aligned}
h(t, \tau) & =\sum_{n=-\infty}^{\infty}\left(\sum_{j=1}^{N_{\mathrm{S}}} \sum_{m=-\infty}^{\infty} c_{j_{n}} e^{\left(\left(\eta_{j}+\imath n \Omega\right)(t-\tau)\right)} l_{j_{m}} e^{\imath(n+m) \Omega \tau}\right) \\
& +\sum_{k=-\infty}^{\infty} d_{k} e^{\imath k \Omega t} \delta(t-\tau),
\end{aligned}
$$

where the product $\boldsymbol{L}_{j}^{T}(\tau) \mathbf{B}(\tau)$ and $\mathbf{D}(\tau)$ have been expanded in Fourier series, $l_{j_{m}}$ and $d_{k}$ being the related amplitudes. After some manipulations (see also Wereley, 1991; Wereley and Hall, 1990), the Laplace transformation of $h_{k}(t-$ $\tau) \mathrm{e}^{-\ln \Omega(t-\tau)}$ can be finally written as

$H_{k}(s+\imath n \Omega)=\sum_{j=1}^{N_{\mathrm{s}}} \sum_{m=-\infty}^{\infty} \frac{c_{j_{k+m}} l_{j-m}}{s-\left(\eta_{j}+\imath(m-n) \Omega\right)}+d_{k}$.
Consider now the row index $\ell \in \mathbb{Z}$ and the column index $r \in \mathbb{Z}$ of the HTF, defined such that the element with $\ell=$ $r=0$ (noted as $G_{0,0}$ ) corresponds to the median element $H_{0}(s)$ and the element with $\ell=r=-1$ (noted as $G_{-1,-1}$ ) to $H_{0}(s-i \Omega)$. Hence, according to such definitions and thanks to Eq. (A42), the following holds:

$$
\begin{aligned}
G_{\ell, r}(s) & =H_{\ell-r}(s+\imath r \Omega) \\
& =\sum_{j=1}^{N_{\mathrm{S}}} \sum_{w=-\infty}^{\infty} \frac{c_{j \ell+w} l_{j-r-w}}{s-\left(\eta_{j}+\imath w \Omega\right)}+d_{\ell-r} .
\end{aligned}
$$

Consequently, the HTF can be computed as

$\mathcal{G}(s)=\sum_{j=1}^{N_{\mathrm{s}}} \sum_{w=-\infty}^{\infty} \frac{\overline{\mathbf{C}}_{j, w} \overline{\mathbf{B}}_{j, w}^{T}}{s-\left(\eta_{j}+\imath w \Omega\right)}+\overline{\mathbf{D}}$,

where

$\overline{\mathbf{C}}_{j, r}=\left(\begin{array}{lllll}\cdots & c_{j_{-1+w}} & c_{j_{w}} & c_{j_{1+w}} & \cdots\end{array}\right)^{T}$,

$\overline{\mathbf{B}}_{j, m}=\left(\begin{array}{lllll}\cdots & l_{j_{1-w}} & l_{j_{-w}} & l_{j_{-1-w}} & \cdots\end{array}\right)^{T}$,

and $\overline{\mathbf{D}}=\mathcal{D}$.

From a practical standpoint, the use of the harmonic inputoutput relation expressed by the HTF implies that one has to consider a truncated finite dimensional approximation of $\mathcal{G}(s)$, which corresponds to the use of truncated versions of the EMP input and output signals. The convergence of truncated HTFs has been discussed in Sandberg et al. (2005).

\section{A3 The discrete-time case}

In this section the stability analysis of periodic discrete-time systems is briefly reviewed. For a more comprehensive treatment, the reader is referred to Bittanti and Colaneri (2009) and Bottasso and Cacciola (2015).

The autonomous dynamic equation of a generic LTP system in discrete time and its initial conditions are

$\boldsymbol{x}(k+1)=\mathbf{A}(k) \boldsymbol{x}(k), \quad \boldsymbol{x}(0)=\boldsymbol{x}_{0}$,

where $k$ is a generic time instant and $\mathbf{A}(k)$ is a periodic matrix of period $K$ such that $\mathbf{A}(k+K)=\mathbf{A}(k), \forall k$. Similarly, the transition matrix obeys the following equation with its initial conditions:

$\boldsymbol{\Phi}(k+1, \kappa)=\mathbf{A}(k) \boldsymbol{\Phi}(k, \kappa), \quad \boldsymbol{\Phi}(\kappa, \kappa)=\mathbf{I}$.

In this work we consider only reversible systems, i.e., those for which $(\boldsymbol{\Phi}(k, \kappa)) \neq 0, \forall(k, \kappa)$.

For reversible discrete-time systems, the state transition matrix $\boldsymbol{\Phi}(k, \kappa)$ can be decomposed into periodic and contractive parts as

$\boldsymbol{\Phi}(k, \kappa)=\mathbf{P}(k) \mathbf{R}^{(k-\kappa)} \mathbf{P}(\kappa)^{-1}$, 
where $\mathbf{P}(k)$ is periodic and $\mathbf{R}$ is constant. Here again, the system is stable if the characteristic multipliers $\theta_{j}$, i.e., the eigenvalues of the monodromy matrix $\boldsymbol{\Psi}(\kappa)=$ $\mathbf{P}(\kappa) \mathbf{R}^{(K)} \mathbf{P}(\kappa)^{-1}$, belong to the open unit disk in the complex plane. The relationship between characteristic multipliers and characteristic exponents is

$\theta_{j}=\eta_{j}^{K}$.

In the discrete-time case, the apparent multiplicity of the characteristic exponents manifests itself as a phase indetermination since

$\eta_{j}=\sqrt[K]{\left|\theta_{j}\right|} \exp \left(l \frac{L\left(\theta_{j}\right)+2 \ell \pi}{K}\right)$,

where $\ell=0, \ldots, K-1$ is an arbitrary integer. As in the continuous-time case, this does not in reality generate any inconsistency as frequencies, damping, and participation factors of the various harmonics are unaffected by this apparent arbitrariness.

Following the same approach of the continuous-time case, the transition matrix can be rewritten as

$\boldsymbol{\Phi}(k, \kappa)=\sum_{j=1}^{N_{\mathrm{s}}} \boldsymbol{\psi}_{j}(k) \boldsymbol{L}_{j}(\kappa)^{T} e^{\hat{\eta}_{j}(k-\kappa)}$,

where $\mathbf{\Xi}(k)=\mathbf{P}(k) \mathbf{V}$ and

$$
\begin{aligned}
\boldsymbol{\psi}_{j}(k) & =\operatorname{col}_{j}(\boldsymbol{\Xi}(k)), \\
\boldsymbol{L}_{j}(\kappa)^{T} & =\operatorname{row}_{j}\left(\boldsymbol{\Xi}^{-1}(\kappa)\right) .
\end{aligned}
$$

After having expanded $\psi_{j}(k)$ in Fourier series, one gets

$$
\begin{aligned}
& \boldsymbol{\Phi}(k, \kappa)=\sum_{j=1}^{N_{\mathrm{S}}} \sum_{n=0}^{K-1} \boldsymbol{\psi}_{j_{n}} \boldsymbol{L}_{j}(\kappa)^{T} \\
& \left(\left|\hat{\eta}_{j}\right| \exp \left(\iota\left(\angle\left(\hat{\eta}_{j}\right)+n \frac{2 \pi}{K}\right)\right)\right)^{k-\kappa},
\end{aligned}
$$

where $\boldsymbol{\psi}_{j_{n}}$ is now the amplitudes of the harmonics of the Fourier expansion of $\boldsymbol{\psi}_{j}(k)$. Coherently, the multiplication of Eq. (A54) with $\mathbf{C}(k)$ leads to

$$
\begin{aligned}
& \boldsymbol{\Phi}_{y}(k, \kappa)=\sum_{j=1}^{N_{\mathrm{s}}} \sum_{n=0}^{K-1} c_{j_{n}} \boldsymbol{L}_{j}(\kappa)^{T} \\
& \left(\left|\hat{\eta}_{j}\right| \exp \left(\iota\left(\angle\left(\hat{\eta}_{j}\right)+n \frac{2 \pi}{K}\right)\right)\right)^{k-\kappa},
\end{aligned}
$$

$c_{j_{n}}$ being the harmonics of the Fourier expansion of $\mathbf{C}(k) \boldsymbol{\psi}_{j}(k)$. This shows that the $j$ th mode is characterized by $K$ exponents with the same modulus and different phases. Each exponent can be transformed into the continuous one using the following expression (cf. Franklin and Powell, 1980):

$\eta_{j_{c}}=\frac{1}{\Delta t} \ln \left(\eta_{j_{d}}\right)$, where $\Delta t$ is the sampling time and subscripts $(\cdot)_{c}$ and $(\cdot)_{d}$ refer, respectively, to the continuous- and discrete-time cases. Once the continuous-time exponents are computed, frequencies, damping, and participation factors can be readily obtained as in the continuous-time case.

\section{Appendix B: Derivation of the equations of motion for a wind turbine analytical model}

The simplified upwind horizontal-axis wind turbine model used in this work, depicted in Fig. B1, considers the coupled motion of tower and blades. The tower fore-aft and sideside flexibility are rendered by two equivalent linear springs and dampers. Each blade is modeled as two rigid bodies connected to each other by means of two equivalent revolute joints, which allow, respectively, the blade flap and edgewise rotations. The inner part of the blade is rigidly connected to the hub. Each joint is associated with a rotational spring and a rotational damper. The inertial and structural characteristics of each element are chosen so as to match the first tower fore-aft and side-side mode and the first blade flapwise and edgewise modes in vacuo, computed using a highfidelity multi-body model of the wind turbine.

The reference frame used for the derivation of the equations of motions has its origin located at the hub, the $x$ axis directed downward, the $z$ axis directed from the tower to the rotor, and the $y$ axis selected so as to form a right-handed triad. To simplify the notation, in the following subscript $k$, denoting the blade number, will be dropped together with the time dependence whenever possible.

The contribution of the two blade parts to the total energy can be developed separately. Thus, let $r_{U}$ and $r_{D}$ indicate, respectively, the dimensional abscissa along the inner and the movable parts of the blade. The position of a generic blade point is given by

$\boldsymbol{r}_{U}=\left(\begin{array}{c}r_{U} \cos \psi \\ y_{H}+r_{U} \sin \psi \\ z_{H}\end{array}\right)$

when the point belongs to the inner part of the blade and by

$\boldsymbol{r}_{D}=\left(\begin{array}{c}e \cos \psi+r_{D} \cos \beta \cos (\psi+\zeta) \\ y_{H}+e \sin \psi+r_{D} \cos \beta \sin (\psi+\zeta) \\ z_{H}+r \sin \beta\end{array}\right)$

when it belongs to the movable part. The kinetic energy of the whole rotor is obtained by summing up the kinetic energy of the hub, $T_{H}$, and of both the inner and the movable parts of the $k$ th blade, respectively, noted as $T_{D_{k}}$ and $T_{U_{k}}$, resulting in

$T=T_{H}+\sum_{k=1}^{B}\left(T_{U_{k}}+T_{D_{k}}\right)$,

where

$T_{H}=\frac{1}{2} m_{\mathrm{H}}\left(\dot{y}_{H}^{2}+\dot{z}_{H}^{2}\right)$ 
Structural properties and degrees of freedom

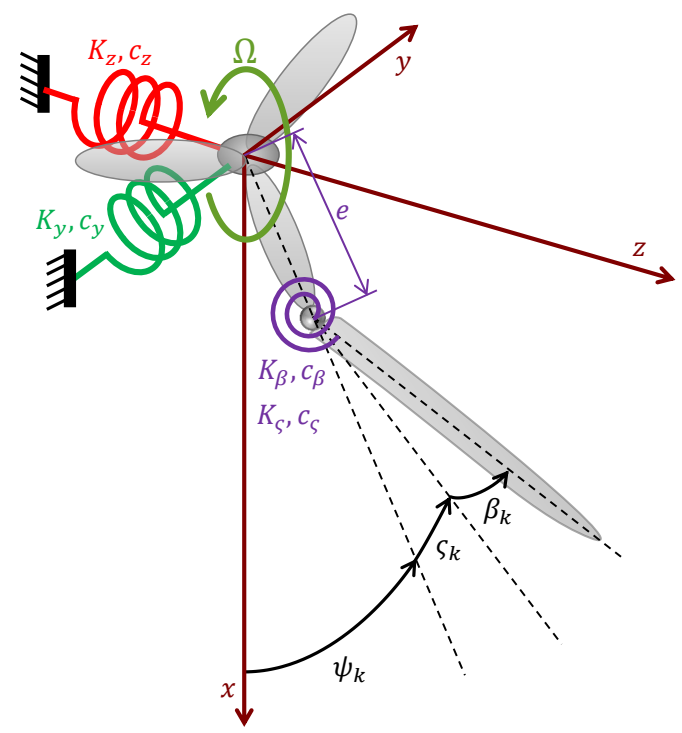

Inertial properties

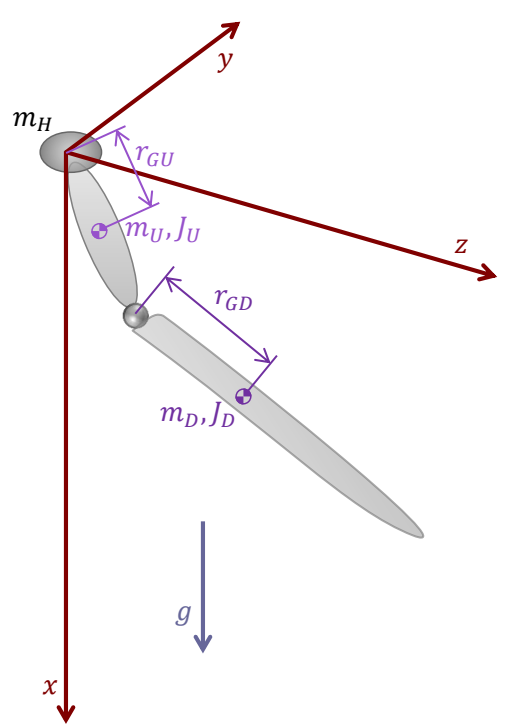

Figure B1. Sketch of the wind turbine analytical model. Only one blade is shown in order to avoid cluttering the figure.

and

$T_{U_{k}}=\frac{1}{2} \int_{0}^{e} \rho(r) \dot{\boldsymbol{r}}_{U}(r) \cdot \dot{\boldsymbol{r}}_{U}(r) \mathrm{d} r$

$T_{D_{k}}=\frac{1}{2} \int_{e}^{R} \rho(r) \dot{\boldsymbol{r}}_{D}(r) \cdot \dot{\boldsymbol{r}}_{D}(r) \mathrm{d} r$

$\rho(r)$ being the blade mass per unit span.

All springs and gravity contribute to the potential energy of the system as

$V=V_{y_{H}}+V_{z_{H}}+\sum_{k=1}^{B}\left(V_{\beta_{k}}+V_{\zeta_{k}}+V_{U_{k}}+V_{D_{k}}\right)$,

where the potential energy of the side-side and fore-aft springs is defined, respectively, as $V_{y_{H}}=1 / 2 K_{y} y_{H}^{2}$ and $V_{z_{H}}=1 / 2 K_{z} z_{H}^{2}$, while that of the flap-wise and edgewise springs is defined as $V_{\beta_{k}}=1 / 2 K_{\beta} \beta_{k}^{2}$ and $V_{\zeta_{k}}=1 / 2 K_{\zeta} \zeta_{k}^{2}$. Finally the contribution of gravity can be expressed as

$V_{U}=-m_{U} g x_{C G_{U}}=-m_{U} g r_{G U} \cos \psi$,

$V_{D}=-m_{D} g x_{C G_{D}}=-m_{D} g r_{\mathrm{GD}} \cos \beta \cos (\psi+\zeta)$.

The damping function $D$ follows a rather similar procedure, where

$D=D_{y_{H}}+D_{z_{H}}+\sum_{k=1}^{B}\left(D_{\beta_{k}}+D_{\zeta_{k}}\right)$.

The aerodynamic model is based on a linearized BEM approach with constant aerodynamic properties along the
Table B1. Definitions of the symbols in the aerodynamic loads.

\begin{tabular}{lll}
\hline Symbol & Meaning & Expression \\
\hline$\theta_{p}$ & Pitch angle & \\
$K_{1}$ & Vertical shear gradient & \\
$U_{0}, V_{0}$ & Cross and axial wind & \\
$v_{i}$ & Axial induced velocity & \\
$\lambda$ & Nondimensional inflow & $\left(V_{0}-v_{i}-\dot{z}_{H}\right) /(\Omega R)$ \\
$\bar{U}_{0}$ & Nondimensional cross-flow & $\left(U_{0}-\dot{y}_{H}\right) /(\Omega R)$ \\
$\bar{V}_{0}$ & Nondimensional axial wind & $V_{0} /(\Omega R)$ \\
$\gamma$ & Lock number & $\rho C_{l_{\alpha}} c R^{4} / J_{D}$ \\
\hline
\end{tabular}

blade, mostly taken from Eggleston and Stoddard (1987), with the addition of the hub velocity $\left(\dot{y}_{H}, \dot{z}_{H}\right)$ to the inflow and cross-flow terms but neglecting the yaw rate. Table B1 gives the meaning of some symbols used in the following equations.

The hub shear force in the fore-aft direction is

$$
\begin{aligned}
S_{\beta}^{\text {aero }} & =\frac{1}{2} \gamma J_{D} \frac{\Omega^{2}}{R}\left\{\frac{\lambda}{2}+\frac{\theta_{p}}{3}-\frac{\dot{\beta} / \Omega}{3}-\sin \psi\left[\frac{\bar{U}_{0} \beta}{2}\right]\right. \\
& \left.-\cos \psi\left[\bar{U}_{0}\left(\lambda-\frac{\dot{\beta} / \Omega}{2}-\theta_{p}\right)+\frac{K_{1} \bar{V}_{0}}{3}\right]\right\} .
\end{aligned}
$$

The hinge out-of-plane moment is

$$
\begin{aligned}
M_{\beta}^{\text {aero }} & =\frac{1}{2} \gamma J_{D} \Omega^{2}\left\{\frac{\lambda}{3}+\frac{\theta_{p}}{4}-\frac{\dot{\beta} / \Omega}{4}-\sin \psi\left[\frac{\bar{U}_{0} \beta}{3}\right]\right. \\
& \left.-\cos \psi\left[\bar{U}_{0}\left(\frac{\lambda}{2}-\frac{\dot{\beta} / \Omega}{3}+\frac{2 \theta_{p}}{3}\right)+\frac{K_{1} \bar{V}_{0}}{4}\right]\right\} .
\end{aligned}
$$


The hub shear force in the direction parallel to the chord of the blade, and pointing towards the leading edge, is

$$
\begin{aligned}
& S_{\zeta}^{\text {aero }}=\frac{1}{2} \gamma J_{D} \frac{\Omega^{2}}{R}\left\{\lambda\left(\lambda+\frac{\theta_{p}}{2}\right)-\frac{\dot{\beta}}{\Omega}\left(\lambda+\frac{\theta_{p}}{3}\right)\right. \\
& -\cos \psi\left[K_{1} \bar{V}_{0}\left(\lambda+\frac{\theta_{p}}{3}\right)+\bar{U}_{0} \theta_{p} \lambda-\frac{\dot{\beta}}{\Omega}\left(\frac{2}{3} K_{1} \bar{V}_{0}+\frac{\bar{U}_{0} \theta_{p}}{2}\right)\right] \\
& \left.-\sin \psi\left[\beta \bar{U}_{0}\left(2 \lambda+\frac{\theta_{p}}{2}-\frac{\dot{\beta}}{\Omega}\right)\right]\right\} .
\end{aligned}
$$

The hinge moment in the edgewise direction is

$$
\begin{aligned}
M_{\zeta}^{\text {aero }} & =\frac{1}{2} \gamma J_{D} \Omega^{2}\left\{\lambda\left(\frac{\lambda}{2}+\frac{\theta_{p}}{3}\right)-\frac{\dot{\beta}}{\Omega}\left(\frac{2}{3} \lambda+\frac{\theta_{p}}{4}\right)\right. \\
& -\cos \psi\left[K_{1} \bar{V}_{0}\left(\frac{2}{3} \lambda+\frac{\theta_{p}}{4}\right)+\frac{\bar{U}_{0} \theta_{p} \lambda}{2}\right. \\
& \left.-\frac{\dot{\beta}}{\Omega}\left(\frac{K_{1} \bar{V}_{0}}{2}+\frac{\bar{U}_{0} \theta_{p}}{3}\right)\right] \\
& \left.-\sin \psi\left[\beta \bar{U}_{0}\left(\lambda+\frac{\theta_{p}}{3}-\frac{2}{3} \frac{\dot{\beta}}{\Omega}\right)\right]\right\} .
\end{aligned}
$$

This aerodynamic model assumes that the wind velocity varies linearly over the rotor disc, and therefore it is not suited to simulate turbulent wind fields.

The virtual work of the aerodynamic forces and moments results in

$$
\begin{aligned}
\delta W^{\text {aero }} & =\sum_{k=1}^{B}\left(S_{\zeta_{k}}^{\text {aero }} \cos \left(\psi_{k}+\zeta_{k}\right) \delta y_{H}+S_{\beta_{k}}^{\text {aero }} \delta z_{H}\right. \\
& \left.+M_{\beta_{k}}^{\text {aero }} \delta \beta_{k}+M_{\zeta_{k}}^{\text {aero }} \delta \zeta_{k}\right) .
\end{aligned}
$$

The generalized forces follow directly from the previous expression.
Finally, the nonlinear Lagrangian equations of motion of the system are

$$
\begin{aligned}
J_{D} \ddot{\beta} & +C_{\beta} \dot{\beta}+K_{\beta} \beta=M_{\beta}^{\text {aero }}-J_{D}(\Omega+\dot{\zeta})^{2} \cos \beta \sin \beta \\
& -m_{D} r_{\mathrm{GD}}\left(g \cos (\psi+\zeta) \sin \beta+e \Omega^{2} \cos \zeta \sin \beta\right. \\
& \left.-\ddot{y}_{H} \sin (\psi+\zeta) \sin \beta+\ddot{z}_{H} \cos \beta\right), \\
J_{D} \cos ^{2} \beta \ddot{\zeta}+C_{\zeta} \dot{\zeta}+K_{\zeta} \zeta=M_{\zeta}^{\text {aero }}+2 J_{D}(\Omega+\dot{\zeta}) \dot{\beta} \cos \beta \sin \beta & \quad(\mathrm{B} 14 \mathrm{a}) \\
& -m_{D} r_{\mathrm{GD}} \cos \beta(g \sin (\psi+\zeta) \\
& \left.+e \Omega^{2} \sin \zeta+\ddot{y}_{H} \cos (\psi+\zeta)\right), \\
\left(m_{\mathrm{H}}\right. & \left.+B\left(m_{U}+m_{D}\right)\right) \ddot{z}_{H}+C_{z} \dot{z}_{H}+K_{z} z_{H} \\
& =\sum_{k=1}^{B}\left(S_{\beta_{k}}^{\mathrm{aero}}-m_{D} r_{\mathrm{GD}}\left(\ddot{\beta}_{k} \cos \beta_{k}-\dot{\beta}_{k}^{2} \sin \beta\right)\right), \quad(\mathrm{B} 14 \mathrm{c}) \\
\left(m_{\mathrm{H}}\right. & \left.+B\left(m_{U}+m_{D}\right)\right) \ddot{y}_{H}+C_{y} \dot{y}_{H}+K_{y} y_{H} \\
& =\sum_{k=1}^{B}\left(S_{\zeta_{k}}^{\mathrm{aero}} \cos \left(\psi_{k}+\zeta_{k}\right)+m_{D} r_{\mathrm{GD}}\left(\Omega^{2} \cos \beta_{k} \sin \left(\psi_{k}+\zeta_{k}\right)\right.\right. \\
& +\dot{\beta}_{k}^{2} \cos \beta_{k} \sin \left(\psi_{k}+\zeta_{k}\right)+2 \dot{\beta}_{k} \dot{\zeta}_{k} \sin \beta_{k} \cos \left(\psi_{k}+\zeta_{k}\right) \\
& +\dot{\zeta}_{k}^{2} \cos \beta_{k} \sin \left(\psi_{k}+\zeta_{k}+2 \Omega\left(\dot{\beta}_{k} \sin \beta_{k} \cos \left(\psi_{k}+\zeta_{k}\right)\right.\right. \\
& \left.+\dot{\zeta}_{k} \cos \beta_{k} \sin \left(\psi_{k}+\zeta_{k}\right)\right)+\ddot{\beta}_{k} \sin \beta_{k} \sin \left(\psi_{k}+\zeta_{k}\right) \\
& \left.\left.-\ddot{\zeta}_{k} \cos \beta_{k} \cos \left(\psi_{k}+\zeta_{k}\right)\right)\right) .
\end{aligned}
$$

All equations shown in this section and the system linearization were computed analytically with Wolfram Mathematica ${ }^{\circledR}$ (Wolfram Research, 2013). 


\section{Appendix C: Nomenclature}

$\mathfrak{A}(q ; k) \quad$ Periodic autoregressive polynomial

$\mathfrak{B}(q ; k) \quad$ Periodic exogenous polynomial

$N_{a} \quad$ Order of the autoregressive part

$N_{b} \quad$ Order of the exogenous part

$N_{g} \quad$ Order of the moving average part

$\mathfrak{F}(q ; k) \quad$ Shape filter polynomial

$\mathfrak{G}(q ; k) \quad$ Periodic moving average polynomial

$K \quad$ Discrete-time system period

$T \quad$ Continuous-time system period

$J \quad$ Cost function

$N \quad$ Total number of samples used for identification

$N_{\mathrm{S}} \quad$ Number of states

$\mathcal{P}(\boldsymbol{p}) \quad$ Characteristic multipliers of the PARMAX predictor $\boldsymbol{p}$

$N_{F_{a}} \quad$ Number of harmonics of the autoregressive coefficients

$N_{F_{b}} \quad$ Number of harmonics of the exogenous coefficients

$N_{F_{g}} \quad$ Number of harmonics of the moving average coefficients

$\mathbb{C} \quad$ Complex number set

$\mathbb{Z} \quad$ Integer number set

$B \quad$ Number of blades

$Y(s) \quad$ Laplace transformation of the output

$U(s) \quad$ Laplace transformation of the input

$H_{k}(s) \quad$ Laplace transformation of the $k$ th harmonic of the impulse response

$z \quad$ Measured output

$\hat{z} \quad$ Predicted output

$q \quad$ One-step-ahead shift operator

$k \quad$ Time index

$u_{\mathrm{t}} \quad$ Turbulent wind input

$u \quad$ Mean wind speed

$n \quad$ Order of the system, $n=\max \left(N_{a}, N_{b}, N_{g}\right)$

$y \quad$ System output

e Process noise

$y_{k}(\omega) \quad$ Fourier transformation of the $k$ th shifted copy of the output

$u_{k}(\omega) \quad$ Fourier transformation of the $k$ th shifted copy of the input

$y_{k}(s) \quad$ Laplace transformation of the $k$ th shifted copy of the output

$u_{k}(s) \quad$ Laplace transformation of the $k$ th shifted copy of the input

$t \quad$ Time

$y_{\mathrm{F}} \quad$ Forced response

$h(t, \tau) \quad$ Impulse response

$h_{k}(t) \quad k$ th harmonic of the impulse response

$l \quad$ Imaginary unit

$s \quad$ Laplace variable

$\mathbf{A}(t) \quad$ State matrix

$\mathbf{B}(t) \quad$ Input matrix
$\mathbf{E}(t) \quad$ Process noise input matrix

$\mathbf{C}(t) \quad$ Output matrix

$\mathbf{D}(t) \quad$ Direct transition matrix

$\mathbf{F}(t) \quad$ Measurement noise matrix

$\mathbf{N}(t) \quad$ State matrix of the PARMAX predictor

$\mathcal{G} \quad$ Harmonic transfer function

$\mathcal{U} \quad$ Exponentially modulated periodic expansion of the input

$\mathcal{Y} \quad$ Exponentially modulated periodic expansion of the output

$\boldsymbol{Y}(\omega) \quad$ Vector of Fourier transformations of all shifted copies of the output

$\boldsymbol{U}(\omega) \quad$ Vector of Fourier transformations of all shifted copies of the input

$\mathbf{G}(\omega) \quad$ Harmonic frequency response function

$\mathbf{S}_{Y Y}(\omega)$ Harmonic power spectrum of the output

$\mathbf{S}_{U U}(\omega)$ Harmonic power spectrum of the input

$\boldsymbol{\Phi} \quad$ State transition matrix

I Identity matrix

$\Psi \quad$ Monodromy matrix

S Eigenvector matrix of the monodromy matrix

$\mathbf{Q}(t) \quad$ Floquet-Lyapunov transformation

R Floquet factor

V Eigenvector matrix of the Floquet factor

$\mathbf{P}(t) \quad$ Periodic eigenvector

$\boldsymbol{x} \quad$ State vector

$\boldsymbol{p} \quad$ Unknown vector of model coefficients

$\boldsymbol{x}_{0} \quad$ Initial state vector

$\tilde{\boldsymbol{x}}_{\tau} \quad$ State vector sampled at every period

$\boldsymbol{s}_{j} \quad j$ th eigenvector of the monodromy matrix

$z \quad$ Floquet-Lyapunov transformed state vector

$\Omega \quad$ Rotor speed

$\delta_{u_{\mathrm{t}}} \quad$ Turbulent perturbation of the wind

$\alpha_{i} \quad i$ th coefficient of canonical system matrix $\mathbf{A}$

$\beta_{i} \quad i$ th coefficient of canonical input matrix $\mathbf{B}$

$\gamma_{i} \quad i$ th coefficient of canonical process noise input matrix $\mathbf{E}$

$\psi \quad$ Azimuth angle

$\varepsilon \quad$ Prediction error

$\omega \quad$ Generic frequency

$\eta_{j} \quad j$ th characteristic exponent

$\tau \quad$ Time lag

$\theta_{j} \quad j$ th characteristic multiplier

$\phi_{j_{n}} \quad$ Participation factor of the $n$th harmonic of the $j$ th mode

$\phi_{j_{n}}^{y} \quad$ Output-specific participation factor of the $n$th harmonic of the $j$ th mode

$(\cdot)^{*} \quad$ Complex conjugate

$(\cdot)^{\mathrm{s}} \quad$ Sine amplitude

$(.)^{\mathrm{c}} \quad$ Cosine amplitude

$(\cdot)^{\mathrm{T}} \quad$ Transpose

$(\cdot)^{H} \quad$ Complex conjugate transpose

$(\dot{.}) \quad$ Time derivative 


$\begin{array}{ll}\text { IPC } & \text { Individual pitch control } \\ \text { HTF } & \text { Harmonic transfer function } \\ \text { HFRF } & \text { Harmonic frequency response function } \\ \text { MBC } & \text { Multi-blade coordinate } \\ \text { LTI } & \text { Linear time-invariant } \\ \text { LTP } & \text { Linear time periodic } \\ \text { ARMAX } & \text { Autoregressive moving average with exoge- } \\ & \text { nous input } \\ \text { PARMAX } & \text { Periodic ARMAX } \\ \text { POMA } & \text { Periodic operational modal analysis } \\ \text { PEM } & \text { Prediction error method } \\ \text { EMP } & \text { Exponentially modulated periodic } \\ \text { SISO } & \text { Single-input single-output } \\ \text { PSD } & \text { Power spectral density } \\ \text { HPSD } & \text { Harmonic PSD } \\ \text { BEM } & \text { Blade element momentum } \\ \text { SS } & \text { State-space } \\ \text { CG } & \text { Center of gravity }\end{array}$


Edited by: M. Muskulus

Reviewed by: two anonymous referees

\section{References}

Allen, M. S. and Ginsberg, J. H.: Floquet modal analysis to detect cracks in a rotating shaft on anisotropic supports, Proceedings of 24th IMAC Conference, 30 January-2 February 2006, St. Louis, Missouri, 2006.

Allen, M. S., Chauhan, S., and Hansen, M. H.: Advanced operational modal analysis methods for linear time periodic system identification, Proceedings of 29th IMAC Conference, 31 January-3 February 2011, Jacksonville, Florida, doi:10.1007/978-1-4419-9316-8_3, 2011a.

Allen, M. S., Sracic, M. W., Chauhan, S., and Hansen, M. H.: Output-only modal analysis of linear time periodic systems with application to wind turbine simulation data, Mech. Syst. Signal Pr., 25, 1174-1191, doi:10.1016/j.ymssp.2010.12.018, $2011 \mathrm{~b}$.

Avendaño-Valencia, L. D. and Fassois, S. D.: In-operation identification of wind turbine non-stationary dynamics: a comparison of various methods, Proceedings of 5th IOMAC Conference, 1315 May 2013, Guimarães, Portugal, 2013.

Avendaño-Valencia, L. D. and Fassois, S. D.: Stationary and nonstationary random vibration modelling and analysis for an operating wind turbine, Mech. Syst. Signal Pr., 47, 263-285, doi:10.1016/j.ymssp.2013.07.022, 2014.

Bertogalli, V., Bittanti, S., and Lovera, M.: Simulation and identification of helicopter rotor dynamics using a generalpurpose multibody code, J. Franklin I., 366, 783-797, doi:10.1016/S0016-0032(98)00053-2, 1999.

Bittanti, S. and Colaneri, P.: Periodic Systems - Filtering and Control, Springer-Verlag, London, 2009.

Bittanti, S. and De Nicolao, G.: Spectral factorization of linear periodic systems with application to the optimal prediction of periodic ARMA models, Automatica, 29, 517-522, doi:10.1016/0005-1098(93)90149-N, 1993.

Bittanti, S., Bolzern, P., De Nicolao, G., Piroddi, L., and Purassanta, D. A.: Minimum prediction error algorithm for estimation of periodic ARMA models, Proceeding of the ECC 1991 European Control Conference, Grenoble, France, 1200-1203, 1991.

Bittanti, S., Bolzern, P., De Nicolao, L., and Piroddi, L.: Representation, prediction and identification of cyclostationary processes A state space approach, in: Cyclostationarity in Communications and Signal Processing, edited by: Gardner W. A., IEEE Press, Piscataway, NJ, 267-294, 1994.

Borri, M.: Helicopter Rotor Dynamics by Finite Element Time Approximation, Comput. Math. Appl., 12, 149-160, doi:10.1016/0898-1221(86)90092-1, 1986.

Bottasso, C. L. and Cacciola, S.: Model-independent periodic stability analysis of wind turbines, Wind Energy, 18, 865-887, doi:10.1002/we.1735, 2015.

Bottasso, C. L. and Croce, A.: Cp-Lambda: User's Manual, Dipartimento di Scienze e Tecnologie Aerospaziali, Politecnico di Milano, Milano, Italy, 2006-2016.

Bottasso, C. L., Cacciola, S. and Riva, R.: Floquet stability analysis of wind turbines using input-output models, Proceedings of AIAA Scitech - 32nd ASME Wind Energy Symposium, 13-17 January 2014, National Harbor, MD, USA, doi:10.2514/6.2014-0713, 2014.
Byrd, R. H., Hribar, M. E., and Nocedal, J.: An interior point algorithm for large scale nonlinear programming, SIAM J. Optimiz., 9, 8777-900, doi:10.1137/S1052623497325107, 1999.

Byrd, R. H., Gilbert, J. C., and Nocedal, J.: A trust region method based on interior point techniques for nonlinear programming, Math. Program., 89, 149-185, doi:10.1007/PL00011391, 2000.

Carne, T. G. and James III, G. H.: The inception of OMA in the development of modal testing technology for wind turbines, Mech. Syst. Signal Pr., 24, 1213-1226, doi:10.1016/j.ymssp.2010.03.006, 2010.

Chauhan, S., Hansen, M. H., and Tcherniak, D.: Application of operational modal analysis and blind source separation/independent component analysis techniques to wind turbines, Proceedings of 27th IMAC Conference, 9-12 February 2009, Orlando, Florida, 2009.

Coleman, R. P. and Feingold, A. M.: Theory of self-excited mechanical oscillations of helicopter rotors with hinged blades, NACA Report TN 1351, 1958.

Eggleston, D. M. and Stoddard, F. S.: Wind Turbine Engineering Design, Van Nostrand Reinhold, New York, NY, USA, 1987.

Franklin, G. F. and Powell, J. D.: Digital Control of Dynamical Systems, 1st Edn., Addison-Wesley Publishing Company, Reading, MA, USA, 1980.

Hansen, M. H.: Aeroelastic stability analysis of wind turbines using an eigenvalue approach, Wind Energy, 7, 133-143, doi:10.1002/we.116, 2004.

Hansen, M. H., Thomsen, K., and Fuglsang, P.: Two methods for estimating aeroelastic damping of operational wind turbine modes from experiments, Wind Energy, 9, 179-191, doi:10.1002/we.187, 2006.

Hansen, M. O. L., Sørensen, J. N., Voutsinas, S., Sørensen, N., and Madsen, H. A.: State of the art in wind turbine aerodynamics and aeroelasticity, Prog. Aerosp. Sci., 42, 285-330, doi:10.1016/j.paerosci.2006.10.002, 2006.

Hauer, J. F., Demeure, C. J., and Scharf, L. L.: Initial results in Prony analysis of power system response signal, IEEE T. Power Syst., 5, 80-89, doi:10.1109/59.49090, 1990.

Jonkman, B. J. and Kilcher, L.: TurbSim User's Guide: Version 1.06.00, NREL Technical report, 2012.

Kutner, M. H., Nachtsheim, C. J., Neter, J., and Li, W.: Applied Linear Statistical Models, 5th Edn., McGraw-Hill/Irwin, New York, 2005.

Ljung, L.: System Identification - Theory for the User, 2nd Edn., Prentice Hall, Englewood Cliffs, NJ, USA, 1999.

Manwell, J. F., McGowan, J. G., and Rogers, A. L.: Wind Energy Explained - Theory, Design and Application, Wiley, New York, 2009.

Mevel, L., Gueguen, I., and Tcherniak, D.: LPTV subspace analysis of wind turbine data, 7th European Workshop on Structural Health Monitoring, 8-11 July 2014, La Cité, Nantes, France, 221-228, 2014.

Murtagh, P. J. and Basu, B.: Identification of equivalent modal damping for a wind turbine at standstill using Fourier and wavelet analysis, Proceedings of the Institution of Mechanical Engineers, Part K, J. Multi-body Dynam., 221, 577-589, doi:10.1243/14644193JMBD90, 2007.

Peters, D. A. and Lieb, S. M., and Ahaus, L. A.: Interpretation of Floquet eigenvalues and eigenvectors for periodic systems, 
J. Am. Helicopt. Soc., 56, 1-11, doi:10.4050/JAHS.56.032001, 2011.

Rissanen, J.: Algorithms for triangular decomposition of block Hankel and Toeplitz matrices with application to factoring positive matrix polynomials, Math. Comput., 27, 247-154, doi:10.1090/S0025-5718-1973-0329235-5, 1973.

Sandberg, H., Möllerstedt, B., and Bernhardsson, B.: Frequencydomain analysis of Linear Time Periodic Systems, IEEE T. Automat. Contr., 50, 1971-1983, doi:10.1109/TAC.2005.860294, 2005.

Sayed, A. H. and Kailath, T.: A survey of spectral factorization methods, Numer. Lin. Algebr., 8, 467-496, doi:10.1002/nla.250, 2001.

Shifei, Y. and Allen, M. S.: Output-only modal analysis using continuous scan laser doppler vibrometry and application to a $20 \mathrm{~kW}$ wind turbine, Mech. Syst. Signal Pr., 31, 228-245, doi:10.1016/j.ymssp.2012.04.012, 2012.

Shifei, Y. and Allen, M. S.: Lifting approach to simplify outputonly continuous-scan laser vibrometry, Mech. Syst. Signal Pr., 45, 267-282, doi:10.1016/j.ymssp.2013.11.010, 2014.

Skjoldan, P. F. and Bauchau, O. A.: Determination of modal parameters in complex nonlinear systems, J. Comput. Nonlin. Dynam., 6.3, 031017, doi:10.1115/1.4002975, 2011.

Skjoldan, P. F. and Hansen, M. H.: On the similarity of the Coleman and Lyapunov-Floquet transformations for modal analysis of bladed rotor structures, J. Sound Vibrat., 327, 424-439, doi:10.1016/j.jsv.2009.07.007, 2009.

Skjoldan, P. F. and Hansen, M. H.: Implicit Floquet analysis of wind turbines using tangent matrices of a non-linear aeroelastic code, Wind Energy, 15, 275-287, doi:10.1002/we.467, 2012.
Skjoldan, P. F. and Hansen, M. H.: Effects of extreme wind shear on aeroelastic modal damping of wind turbines, Wind Energy, 16, 401-415, doi:10.1002/we.1495, 2013.

Spiridonakos, M. D. and Fassois, S. D.: FS-TARMA models for non-stationary vibration analysis: an overview and comparison, 15th IFAC Symposium on System Identification, 68 July 2009, Saint Malo, France, doi:10.3182/20090706-3-fr2004.00206, 2009.

Tcherniak, D., Chauhan, S., and Hansen, M. H.: Applicability limits of operational modal analysis to operational wind turbines, Proceedings of 28th IMAC Conference, 1-4 February 2010, Jacksonville, Florida, doi:10.1007/978-1-4419-9716-6_29, 2010.

Thomsen, K., Petersen, J. T., Nim, E., Øye, S., and Petersen, B.: A method for determination of damping for edgewise blade vibrations, Wind Energy, 3, 233-246, doi:10.1002/we.42, 2000.

Waltz, R. A., Morales, J. L., Nocedal, J., and Orban, D.: An interior algorithm for nonlinear optimization that combines line search and trust region steps, Math. Program., 107, 391-408, doi:10.1007/s10107-004-0560-5, 2006.

Wereley, N. M.: Analysis and control of linear periodically time varying systems, $\mathrm{PhD}$ thesis, Department of Aeronautics and Astronautics, Massachusetts Institute of Technology, Cambridge, 1991.

Wereley, N. M. and Hall, S. R.: Frequency response of linear time periodic systems, Proceedings of the 29th IEEE Conference on Decision and Control, Honolulu, USA, 3650-3655, doi:10.1109/CDC.1990.203516, 1990.

Wolfram Research: Mathematica, Version 9.0, Wolfram Research, Inc., Champaign, IL, 2013. 